PREPARED FOR THE U.S. DEPARTMENT OF ENERGY, UNDER CONTRACT DE-AC02-76CH03073

PPPL-3640

PPPL-3640

UC-70

Magnetic Field Generation and Electron Acceleration in Relativistic Laser Channel

by

I.Yu. Kostyukov, G. Shvets, N.J. Fisch, and J.M. Rax

December 2001

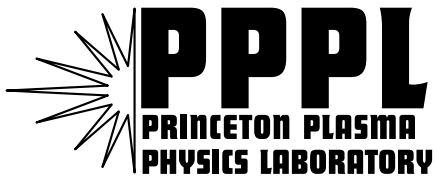

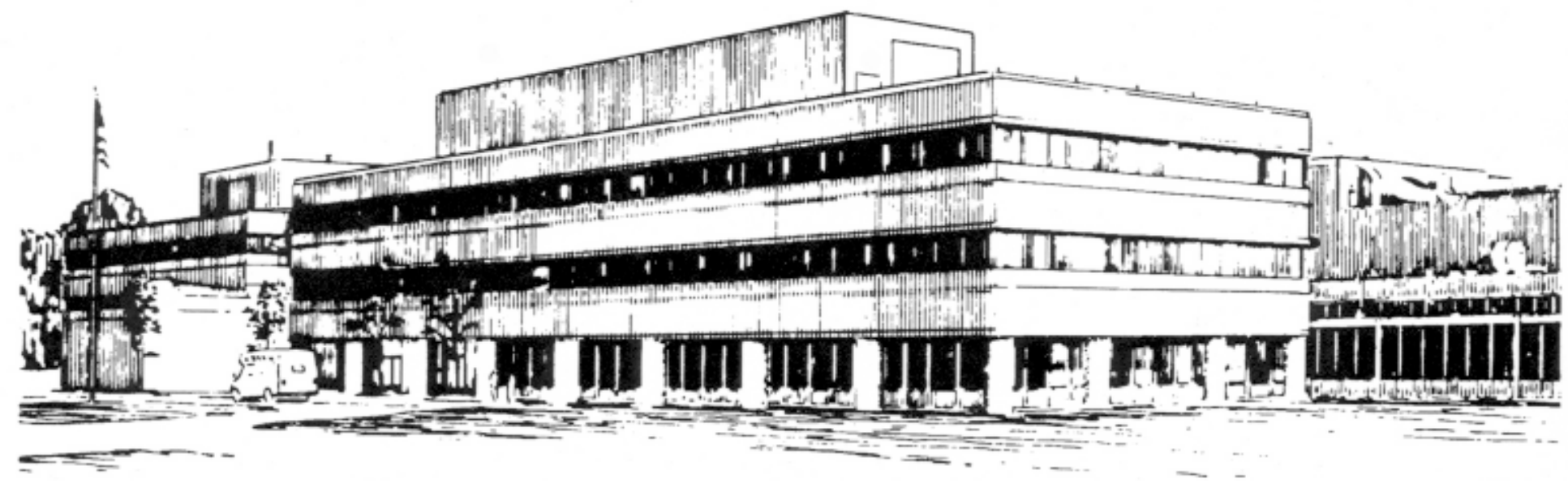

PRINCETON PLASMA PHYSICS LABORATORY PRINCETON UNIVERSITY, PRINCETON, NEW JERSEY 


\section{PPPL Reports Disclaimer}

This report was prepared as an account of work sponsored by an agency of the United States Government. Neither the United States Government nor any agency thereof, nor any of their employees, makes any warranty, express or implied, or assumes any legal liability or responsibility for the accuracy, completeness, or usefulness of any information, apparatus, product, or process disclosed, or represents that its use would not infringe privately owned rights. Reference herein to any specific commercial product, process, or service by trade name, trademark, manufacturer, or otherwise, does not necessarily constitute or imply its endorsement, recommendation, or favoring by the United States Government or any agency thereof. The views and opinions of authors expressed herein do not necessarily state or reflect those of the United States Government or any agency thereof.

\section{Availability}

This report is posted on the U.S. Department of Energy's Princeton Plasma Physics Laboratory Publications and Reports web site in Fiscal Year 2002. The home page for PPPL Reports and Publications is: http://www.pppl.gov/pub_report/

DOE and DOE Contractors can obtain copies of this report from:

U.S. Department of Energy

Office of Scientific and Technical Information

DOE Technical Information Services (DTIS)

P.O. Box 62

Oak Ridge, TN 37831

Telephone: (865) 576-8401

Fax: (865) 576-5728

Email: reports@adonis.osti.gov

This report is available to the general public from:

National Technical Information Service

U.S. Department of Commerce

5285 Port Royal Road

Springfield, VA 22161

Telephone: 1-800-553-6847 or

(703) 605-6000

Fax: (703) 321-8547

Internet: http://www.ntis.gov/ordering.htm 


\title{
Magnetic field generation and electron acceleration in relativistic laser channel
}

I.Yu. Kostyukov

Institute of Applied Physics, Russian Academy of Science, 46 Uljanov St. 603950 Nizhny Novgorod, Russia

G. Shvets and N. J. Fisch

Princeton Plasma Physics Laboratory, Princeton, New Jersey 08543

J. M. Rax

LPMI, Ecole Polytechique, 212, Palaiseau Cedex 91128, France

\begin{abstract}
The interaction between energetic electrons and a circularly polarized laser pulse inside an ion channel is studied. Laser radiation can be resonantly absorbed by electrons executing betatron oscillations in the ion channel and absorbing angular momentum from the laser. The absorbed angular momentum manifests itself as a strong axial magnetic field (inverse Faraday effect). The magnitude of this magnetic field is calculated and related to the amount of the absorbed energy. Absorbed energy and generated magnetic field are estimated for the small and large energy gain regimes. Qualitative comparisons with recent experiments are also made.
\end{abstract}




\section{INTRODUCTION}

The interaction between intense laser radiation and matter is known to produce a wealth of nonlinear effects. Those include fast electron and ion generation $[1,2,3,4,5]$, indicating that ultra-strong electric fields are produced in the course of the laser-plasma interaction. An equally ubiquitous, although less studied, effect accompanying laser-matter interaction is the generation of ultra-strong magnetic fields in the plasma $[6,7,8,9$, 10]. Magnetic fields can have a significant effect on the overall nonlinear plasma dynamics. Extremely high (megagauss) magnetic fields play an essential role in the particle transport, propagation of laser pulses, laser beam self-focusing and penetration of laser radiation into the overdense plasma.

There are several well-understood mechanisms of magnetic field generation (see, for example, Ref. [7] for a review). In many instances, magnetic field is generated by a jet of fast electrons in the direction of laser propagation [6] or by the nonlinear current of the background plasma electrons [8, 11]. Electron currents producing the magnetic field can be viewed as generated due to the momentum transfer from the laser pulse to the plasma electrons. Laser photons carry momentum in the direction of their propagation regardless of their polarization. Therefore, the resulting magnetic field is azimuthal (taking the direction of the laser propagation as a $z$-axis). In this paper we

consider a very different, polarization-dependent method of generating the axial magnetic field.

The generation of the axial magnetic field in the plasma by a circularly (or elliptically) polarized laser is often referred to as the Inverse Faraday Effect (IFE). First theoretically described by Pitaevskii [12] and Steiger and Woods [13], it results from the specifics of the electron motion in a circularly polarized electromagnetic wave. During the interaction of the plasma electrons with the circularly polarized laser pulse, electrons absorb not only the laser energy but also the proportional amount of the total angular momentum of the laser pulse. This angular momentum transfer leads to the electron rotation and generation of the axial magnetic field by the azimuthal electron current. Naturally, IFE is impossible for a linearly polarized laser pulse since it does not possess any angular momentum.

IFE has since been measured in several experiments $[9,14,15]$. The conditions under which IFE is possible are still not fully explored. What is theoretically known [16] is that there is no magnetic field generation during 
the interaction of the inhomogeneous circularly polarized electromagnetic waves with the homogeneous plasma. Magnetic field can be produced in the presence the strong plasma inhomogeneity [17, 18, 19], either pre-formed or developed self-consistently during the interaction.

Here we consider an alternative mechanism of magnetic field generation which involves the resonant energy (and angular momentum) exchange between the laser and the plasma electrons. To our knowledge, this is the first calculation, which explicitly relates the energy deposition by the laser pulse to the magnitude of the magnetic field using a concrete example of the resonance. The resonance occurs between the fast electrons, executing transverse (betatron) oscillations in a fully or partially evacuated plasma channel, and the electric field of the laser pulse. The betatron oscillations are caused by the action of the electrostatic force of the channel ions and self-generated magnetic field. This type of resonant interaction was recently suggested as a mechanism for accelerating electrons to highly-relativistic energies [20, 21]. When a circularly polarized laser pulse is employed, its angular momentum can be transferred to fast resonant electrons along with its energy. The resulting electron beam spirals around the direction of the laser propagation, generating the axial magnetic field [22]. In this paper we calculate the intensity of magnetic field generated in relativistic laser channel taking into account self-generated static field, which is an important extension of known IFE theory $[17,18,19]$. Our calculation is also motivated by the recent experiments at the Rutherford Appleton Laboratory (RAL) [15] which exhibited very large (several megagauss) axial magnetic fields during the propagation of a sub-picosecond laser pulse in a tenuous plasma. The intriguing aspect of the RAL experiment is that both fast electrons and the strong magnetic field were measured in the same experiment. It should be noted that magnetic field generation mechanism firstly proposed in Ref. 22 and considered here has been observed in recent numerical simulation [15].

The basic mechanism of the plasma channel formation is the ponderomotive expulsion of the plasma electrons by the laser pulse. Channel formation in underdense plasma has been confirmed by both experiments [23, 24] and Particle-In-Cell (PIC) simulations [25, 26, 27]. Assuming a circularly polarized Gaussian laser pulse propagating along $z$ axis with vector potential

$$
\mathbf{A}(\mathbf{r}, t)=A_{0} \exp \left(-r^{2} / R^{2}-\xi_{2}^{2} / T^{2}\right)\left[\mathbf{e}_{x} \sin \left(\xi_{1}\right)+\mathbf{e}_{y} \cos \left(\xi_{1}\right)\right],
$$

where $\xi_{1}=\omega t-\omega z / v_{p h}, \xi_{2}=z / v_{g r}-t, v_{p h}$ and $v_{g r}$ are the phase and group 
velocity of the pulse, respectively, the radial ponderomotive force acting on a given is given by

$$
F_{\text {pon }}=-\frac{r}{R^{2}} \frac{e^{2} A_{0}^{2} \exp \left(-2 r^{2} / R^{2}-2 \xi_{2}^{2} / T^{2}\right)}{4 \gamma m c^{2}} .
$$

Here $e$ and $m$ are the charge and the rest mass of electron, $c$ is the speed of light, $\gamma=\sqrt{1-v^{2} / c^{2}}$ is the relativistic gamma factor of the electron. As plasma electrons are expelled by the ponderomotive force, the ion channel forms along the $z$ axis. The radius of the ion channel $r_{c h}$ is estimated by balancing the ponderomotive force and the ion attracting force $F_{r e s}=4 \pi e^{2} n_{i} r$, where $n_{i}$ is the ion density:

$$
e^{2} A_{0}^{2} \exp \left(-2 r_{c h}^{2} / R^{2}-2 \xi_{2}^{2} / T^{2}\right) \simeq 16 \pi n_{i} \gamma m c^{2} R^{2} .
$$

It is seen from Eq. (2) that the ponderomotive force is reduced for hot electrons with $\gamma \gg 1$. They can then remain inside the channel and execute betatron oscillations with frequency $\Omega_{\beta}$ as they propagate along $z$ axis. Laserelectron energy exchange occurs when the resonance condition between the Doppler-upshifted betatron oscillation and laser field is satisfied: $\Omega_{\beta} /(1-$ $\left.\beta_{z} \beta_{p h}\right)=\omega$. If the laser pulse is circularly polarized, electrons can also resonantly absorb a significant amount of its angular momentum.

The main objective of the paper is to calculate energy and angular momentum exchange through this mechanism and obtain an estimate for the generated magnetic field. It is a further object of the paper to describe formally the electron dynamics. We do not address the question of how the hot electrons got pre-accelerated to become resonant with the laser field. As shown below, the required energy boost is very modest (several $\mathrm{MeV}$ ), and can be accomplished, for example, by the plasma waves [1].

Different electron populations are likely to co-exist in the plasma [28]. Some electrons may leave from the side of the channel [20]. These electron populations will not significantly contribute to the magnetic field generation, and will not be considered here. We focus on the other group of electrons which have a modest energy, and are eventually overtaken by the laser pulse. These electrons are left in the channel after the passage of the laser pulse, participating in the magnetic field generation. Another group of electrons may even overtake the laser pulse, generating magnetic field in front of the laser. Regardless of whether the fast electrons are moving faster or slower 
than the laser pulse, the speeds of the electrons and the pulse are close to the speed of light $c$. Since in most short-pulse experiments the length of the plasma is at most $10-20$ times the laser pulse width [1], fast electrons are likely to stay within a few pulse widths from the center of the laser pulse.

The paper is organized as follows. In Section II we describe the electron dynamics in the ion channel in the absence of the laser pulse. By analogy with the quantum mechanical description of a particle in a centrosymmetric potential, we characterize the transverse electron motion using two principle numbers: $I$ (proportional to the total transverse energy) and $L$ (proportional to the $z$ component of the angular momentum). We then calculate the magnetic field and flux produced by an ensemble of electrons with different values of $L$ and $I$. In Section III we describe the coupling between the laser field and electrons and derive a kinetic equation for the electron distribution function $f(I, L)$. Interaction with the laser pulse modifies the electron distribution function, resulting in the energy absorption $Q$ and angular momentum absorption $\Delta L$. The latter produces the magnetic flux $\Phi$ and the averaged over the beam radius magnetic field $B_{\text {ind }}$. In Section IV we calculate $Q, \Phi$, and $B_{\text {ind }}$ in the quasi-linear and strongly nonlinear regimes. The strongly nonlinear and quasi-linear regimes differ from each other by, respectively, large and small distortion of the distribution function. Section V concludes and summarizes the obtained results, making connection with the experimental observations.

\section{ELECTRON DYNAMICS IN THE ION CHANNEL}

An electron in a partially evacuated ion channel, interacting with a laser pulse, can be described by a relativistic Hamiltonian

$$
H=c \sqrt{m c^{2}+\left(\mathbf{p}_{\perp}+e \mathbf{A} / \mathbf{c}\right)^{2}+p_{z}^{2}}+\frac{m \Omega^{2}\left(x^{2}+y^{2}\right)}{2},
$$

where $\Omega^{2}=\omega_{p i}^{2}=4 \pi Z e^{2} n_{i}^{2} / m$. From here on we will use the dimensionless units, normalizing the time to $\omega^{-1}$, the length to $c / \omega$, the momentum to $m c$, and the vector potential and energy to $m c^{2}$. The last term in Hamiltonian (4) describes the electrostatic potential of the channel ions. We assume that the electrons are highly relativistic, $p_{z}^{2} \gg 1+\left(\mathbf{p}_{\perp}+e \mathbf{A}\right)^{2}$. In the highly 
relativistic limit the Lorentz force of the self-generated magnetic field on the electron is almost compensated by the force of the radial electric field of the electron beam [29]. Therefore, only the restoring force of the ions is to remain significant.

For highly relativistic electrons the Hamiltonian (4) can then be expanded as $H \approx H_{0}+H_{\text {int }}$, where $H_{0}$ is the non-interactive Hamiltonian:

$$
H_{0}=p_{z}+\frac{M^{2}}{2 p_{z}}+\frac{p_{\perp}^{2}}{2 p_{z}}+\frac{\epsilon^{2}\left(x^{2}+y^{2}\right)}{2},
$$

and $H_{\text {int }}=e\left(\mathbf{p}_{\perp} \mathbf{A}\right) / p_{z}$ describes the laser-electron interaction. Here $\epsilon=$ $\Omega / \omega$, and $M=\sqrt{m^{2}+e^{2} A^{2} / c^{4}}$ is the renormalized electron mass in a circularly polarized electromagnetic wave. Below, we perform a series of canonical transformation which significantly simplify the non-interactive Hamiltonian $H_{0}$.

\section{A. Canonical transformations}

Since the goal of this calculation is to demonstrate how the angular momentum can be transferred from the laser pulse to the electrons, we proceed by transforming the non-interactive Hamiltonian $H_{0}$ to the form which explicitly contains the electron angular momentum. Introducing cylindrical coordinates $\left(p_{r}, r ; L, \varphi\right)$, the Hamiltonian can be rewritten as

$$
H_{0}=p_{z}+\frac{M^{2}}{2 p_{z}}+\frac{p_{r}^{2}}{2 p_{z}}+\frac{L^{2}}{2 p_{z} r^{2}}+\frac{\epsilon^{2} r^{2}}{2} .
$$

The next step is to introduce the action-angle variables $I=p_{r}^{2} /\left(2 p_{z}\right)+$ $L^{2} /\left(2 p_{z} r^{2}\right)+\epsilon^{2} r^{2} / 2$ and $\theta$ instead of $\left(p_{r}, r\right)$ variables. Here $I$ plays the role of the "principal quantum number", i. e. it is proportional to the total transverse energy. Performing a canonical transformation with the generating function

$$
\begin{aligned}
& S_{1}\left(I, r, L_{1}, \phi, P_{z}, z\right)=P_{z} z+L \varphi+\frac{1}{2} \sqrt{\left(\epsilon \sqrt{P_{z}} r^{2}-I\right)^{2}+L_{1}^{2}-I^{2}} \\
& -\frac{L_{1}}{2} \arcsin \frac{\epsilon \sqrt{P_{z}} I r^{2}-L_{1}^{2}}{r^{2} \epsilon \sqrt{P_{z}} \sqrt{I^{2}-L_{1}^{2}}}-\frac{I}{2} \arcsin \frac{I-r^{2} \epsilon \sqrt{P_{z}}}{\sqrt{I^{2}-L_{1}^{2}}}
\end{aligned}
$$


we obtain the following relationships between the old variables $\left(p_{z}, z ; p_{r}, r\right.$; $L, \varphi)$ and new variables $\left(P_{z}, Z ; I, \theta ; L_{1}, \theta_{L}\right)$ :

$$
\begin{aligned}
p_{z} & =P_{z} \\
L & =L_{1} \\
r^{2} & =\frac{I}{\epsilon \sqrt{P_{z}}}+\frac{\sqrt{I^{2}-L_{1}^{2}}}{\sqrt{P_{z}} \epsilon} \sin (2 \theta) \\
\varphi & =\theta_{L}+\frac{1}{2} \arcsin \frac{\epsilon \sqrt{P_{z}} I r^{2}-L_{1}^{2}}{r^{2} \epsilon \sqrt{P_{z}} \sqrt{I^{2}-L_{1}^{2}}} \\
z & =Z-\frac{\sqrt{I^{2}-L_{1}^{2}}}{4 P_{z}} \cos (2 \theta)
\end{aligned}
$$

where $I$ and $L$ are proportional to the transverse electron energy and the projection of the electron angular momentum on $z$ axis, respectively. Note that $|L| \leq I$, so $L$ can be viewed as the projection of $I$ on the $z$ axis.

Since variables $p_{z}$ and $L$ are not changed as a result of the transformation we will use the old notation $p_{z}$ and $L$ instead of $P_{z}$ and $L_{1}$. In the new variables, the transverse momenta $p_{x}$ and $p_{y}$ are expressed as

$$
\begin{aligned}
& p_{x}=\frac{1}{2}\left[\sqrt{\epsilon \sqrt{p_{z}}(I-L)} \cos \left(\theta-\theta_{L}\right)-\sqrt{\epsilon \sqrt{p_{z}}(I+L)} \sin \left(\theta_{L}+\theta\right)\right], \\
& p_{y}=\frac{1}{2}\left[\sqrt{\epsilon \sqrt{p_{z}}(I+L)} \cos \left(\theta_{L}+\theta\right)-\sqrt{\epsilon \sqrt{p_{z}}(I-L)} \sin \left(\theta-\theta_{L}\right)\right] .
\end{aligned}
$$

The non-interactive Hamiltonian $H_{0}$, expressed in the transformed variables, is given by

$$
H_{0}=p_{z}+\frac{M^{2}}{2 p_{z}}+\frac{\epsilon I}{\sqrt{p_{z}}} .
$$

Note that the longitudinal and transverse degrees of freedom are coupled through the third term in the Eq. (10). The interaction Hamiltonian $H_{\text {int }}$ is expressed in terms of the new canonical variables in Section III. It is also shown in that Section that the interaction term depends on the phase angles $\theta$ and $\theta_{L}$ only as a combination $\left(\theta+\theta_{L}\right)$. This will motivate a further simplifying canonical transformation which emphasizes that the changes in $L$ and $I$ are related. 


\section{B. Electron motion in the channel and magnetic field generation}

Without the interaction term, $I, L$, and $\theta_{L}$ are exact constants, and $\theta$ changes according to

$$
\theta=\frac{\epsilon}{\sqrt{p_{z}}} t
$$

For a vanishing angular momentum $L=0$, Eq. (10) simplifies to

$$
\begin{aligned}
& p_{x}=\sqrt{\epsilon \sqrt{p_{z}} I} \cos (\theta+\pi / 4) \cos \left(\theta_{L}+\pi / 4\right) \\
& p_{y}=\sqrt{\epsilon \sqrt{p_{z}} I} \cos (\theta+\pi / 4) \sin \left(\theta_{L}+\pi / 4\right) .
\end{aligned}
$$

Therefore, electrons with $L=0$ execute linear harmonic oscillations through the origin. The constant angle $\theta_{L}$ defines the orientation of the oscillation direction, and the linearly evolving according to Eq. (11) coordinate $\theta$ marks the oscillation phase. An example of such a trajectory, corresponding to $\theta_{L}=-\pi / 4$, is shown in Fig. 1 as a straight horizontal line. If $L$ is equal to $I$

or $-I$ then the electron performs circular motion with radius $r_{0}=\sqrt{I / \sqrt{p_{z} \epsilon^{2}}}$. In the general case (an arbitrary value of $L$ ) the electron trajectory is an ellipse confined between the maximal radius,

$$
r_{\max }(I, L)=\sqrt{\left(I+\sqrt{I^{2}-L^{2}}\right) / \sqrt{p_{z} \epsilon^{2}}}
$$

and minimal radius

$$
r_{\min }(I, L)=\sqrt{\left(I-\sqrt{I^{2}-L^{2}}\right) / \sqrt{p_{z} \epsilon^{2}}}
$$

Examples of the linear, circular and elliptic orbits are shown in Fig. 1.

Let us consider a group of electrons which are uniformly distributed along the $z$ axis, have the same values of $L=L_{0}$ and $I=I_{0}$, and are evenly distributed over the angles $\theta_{L}, \theta$. It means that electrons uniformly cover the area between the inner circle with radius $r_{\max }$ and the outer circle with radius $r_{\min }$. This group of electrons populate a family of identical elliptical trajectories, each of which is tilted by its own angle $\theta_{L}$. At any given moment in time, electrons are executing their motion along the ellipses according to their phase $\theta$. 
This distribution function $f\left(I, L, \theta, \theta_{L}\right)=\delta\left(I-I_{0}\right) \delta\left(L-L_{0}\right)$ can be projected onto the real space $(r, \varphi)$ by integrating over the phase angles. The corresponding angle-averaged distribution function $F(I, L, r)$ is obtained according to

$$
\begin{aligned}
F(I, L, r)= & \frac{N}{2 \pi} \int_{0}^{2 \pi} \int_{0}^{2 \pi} d \varphi d \theta \frac{1}{r} \delta\left(I-I_{0}\right) \delta\left(L-L_{0}\right) \\
& \times \delta[r-r(I, L, \theta)] \delta[\varphi-\varphi(I, L, \theta)]
\end{aligned}
$$

where $N$ is the linear density of the electrons along the channel and the electron trajectories is taken from Eqs. (8). Integrating over the angles can be performed exactly, yielding

$$
F(I, L, r)=\frac{2 N}{\pi} \frac{\delta\left(I-I_{0}\right) \delta\left(L-L_{0}\right)}{\sqrt{\left[r_{\max }^{2}(I, L)-r^{2}\right]\left[r^{2}-r_{\min }^{2}(I, L)\right]}},
$$

where $r_{\min }(I, L)<r<r_{\max }(I, L)$. Considering a group of electrons with a fixed $I=I_{0}$ which are, in addition, uniformly distributed over the angular momentum $-I_{0}<L_{0}<I_{0}$, the electron distribution function (16) can be integrated over $L, L_{0}$ and $I$ we obtain the electron density in the channel

$$
n(r, \varphi)_{I_{0}}= \begin{cases}\frac{N\left(\epsilon \sqrt{p_{z}}\right)}{2 \pi I_{0}}, & r^{2} \leq \frac{2 I_{0}}{\epsilon \sqrt{p_{z}}} \\ 0, & r^{2}>\frac{2 I_{0}}{\epsilon \sqrt{p_{z}}}\end{cases}
$$

Equation (17) expresses the well-known fact [30] that a uniformly focused beam with a fixed transverse energy ( $I_{0}$, in our case) has a uniform flat-top density distribution in the real space. Despite the singular nature of this, so called, Kapchinskii-Vladimirskii (KV) distribution function, it can be used for analytic estimates.

Using Eq. (16) and integrating over $I$ and $L$ the expression for the azimuthal $(\varphi)$ component of the electron current density in the channel can be derived:

$$
j_{\varphi}=\frac{e N L_{0}}{\pi^{2} r p_{z}} \frac{1}{\sqrt{\left[r_{\max }^{2}\left(I_{0}, L_{0}\right)-r^{2}\right]\left[r^{2}-r_{\min }^{2}\left(I_{0}, L_{0}\right)\right]}} .
$$

Note that the magnitude and the sign of the current depends on $L_{0}$. Clearly, there is no azimuthal current for $L_{0}=0$ because all electrons are executing purely radial oscillations. 
The axial component of static magnetic field can be found from Ampere's law $\nabla \times \mathbf{B}=4 \pi \mathbf{j}$ :

$$
\begin{gathered}
B_{z}(r)=\operatorname{sign}\left(L_{0}\right) \frac{2 e N \Omega}{c} \sqrt{\frac{m c}{p_{z}}} \\
\times \begin{cases}1, & 0<r<r_{\min }, \\
\left(1-\frac{2}{\pi} \arcsin \frac{I_{0} r^{2} \epsilon \sqrt{p_{z}}-L_{0}^{2}}{\left.\epsilon \sqrt{\bar{p}_{z}} r^{2} \sqrt{I_{0}^{2}-L_{0}^{2}}\right),}\right. & r_{\min } \leq r \leq r_{\max }, \\
0, & r>r_{\max } .\end{cases}
\end{gathered}
$$

In writing Eq. () we used the convention $\operatorname{sign}(x)=0$ for $x=0$. This dependence of the magnetic field on the radius $r$ arises because the azimuthal current flows within a cylindrical shell located between $r=r_{\min }$ and $r=r_{\max }$. Magnetic field is uniform inside this "solenoid" if $r<r_{\min }$ and gradually falls off to zero when $r$ is outside of $r_{\max }$. The thickness of the current shell can vary between zero for $\left|L_{0}\right|=I_{0}$ and $r_{\max }$ for $L_{0}=0$.

For future convenience, we also calculate the total magnetic flux $\Phi$ produced by the current shell,

$$
\Phi=\iint \mathbf{B} \cdot d \mathbf{s}=\frac{2 \pi e N L_{0}}{p_{z}} .
$$

and the axial magnetic field $\langle B\rangle$ in the ion channel

$$
\langle B\rangle=\frac{\Phi}{\pi r_{\max }^{2}}=\frac{2 e N \Omega}{c} \sqrt{\frac{m c}{p_{z}}} \frac{L_{0}}{I_{0}+\sqrt{I_{0}^{2}-L_{0}^{2}}} .
$$

It is reasonable to assume that, in the absence of the laser-electron interaction, the electron distribution function $f$ depends only on $I$, i. e. $f \equiv f(I)$. Since an equal number of electrons possess positive and negative angular momenta $L$, the magnetic field produced by such a beam is equal to zero. Interaction with the laser pulse can distort the distribution function and produce a large magnetic field.

\section{INTERACTION BETWEEN ELECTRONS AND LASER FIELD}

The previously introduced interaction Hamiltonian $H_{\text {int }}=e\left(\mathbf{p}_{\perp} \mathbf{A}\right) / p_{z}$ describes the laser-electron interaction. For a Gaussian laser pulse given by 
Eq. (1), using the Bessel function identities

$$
\begin{aligned}
\exp (i y \sin x) & =\sum_{n=-\infty}^{+\infty} J_{n}(y) \exp (i n x) \\
\exp (-y \sin x) & =\sum_{m=-\infty}^{+\infty} I_{m}(y) \exp [i m(x+\pi / 2)]
\end{aligned}
$$

and Eqs. (8), (10), $H_{\text {int }}$ can be expressed as

$$
\begin{aligned}
H_{\text {int }} & =a_{0} \exp \left(-\frac{\xi_{2}^{2}}{T^{2}}-\frac{I}{\epsilon \sqrt{p_{z}} R^{2}}\right) \sum_{m, n=-\infty}^{+\infty} J_{n}\left(\frac{\sqrt{I^{2}-L^{2}}}{4 p_{z}}\right) \\
& \times I_{m}\left(\frac{\sqrt{I^{2}-L^{2}}}{R^{2} \epsilon \sqrt{p_{z}}}\right) \exp [i \theta(m+n)+i m \pi / 2] \\
& \times\left\{\sqrt{\frac{\epsilon(I+L)}{2 p_{z}^{3 / 2}}} \sin \left[\xi_{1}-\theta_{L}-\theta\right]+\sqrt{\frac{\epsilon(I-L)}{2 p_{z}^{3 / 2}}} \cos \left[\xi_{1}-\theta_{L}+\theta\right]\right\},
\end{aligned}
$$

where $a_{0}=e A_{0} /\left(m c^{2}\right)$ is the normalized amplitude of the laser pulse, $J_{n}(x)$ and $I_{m}(x)$ are the Bessel function and the modified Bessel function, respectively.

Bessel functions $J_{m}$ originate from expanding the harmonic part of the vector potential $\vec{A}$ ( $\sin \zeta_{1}$ and $\cos \zeta_{1}$ terms), while the modified $I_{n}$ Bessel functions originate from expanding the $\exp \left(-r^{2} / R^{2}\right)$ term. We will not expand the term $\exp \left(-\xi_{2}^{2} / T^{2}\right)$ because the pulse length is much longer than the amplitude of the electron betatron oscillation in the channel $T v_{g r} \gg$ $\sqrt{\left(I^{2}-L^{2}\right) / p_{z}}$ and harmonic part of this term will be small.

The betatron acceleration of the electron in the ion channel implies that the amplitude of transverse oscillation is less than the radius of the laser channel. Then $I_{0}\left(\frac{\sqrt{I^{2}-L^{2}}}{R^{2} \epsilon \sqrt{p_{z}}}\right) \simeq 1$ and $I_{n \neq 0}\left(\frac{\sqrt{I^{2}-L^{2}}}{R^{2} \epsilon \sqrt{p_{z}}}\right) \simeq 0$. Also, we assume that the argument $\frac{\sqrt{I^{2}-L^{2}}}{4 p_{z}}$ is small, i. e. that the oscillation amplitude in $z$ direction is smaller than $c / \omega$. (This oscillation is caused by the relativistic coupling between the transverse and longitudinal degrees of freedom.) Using these assumptions, we can only retain the interaction term near the fundamental resonance $(m=0, n=0)$ and assume $J_{0}\left(\frac{\sqrt{I^{2}-L^{2}}}{4 p_{z}}\right) \simeq 1, J_{m \neq 0}\left(\frac{\sqrt{I^{2}-L^{2}}}{4 p_{z}}\right) \simeq 0$. Isolating the single most important resonance is a standard approximation technique in the nonlinear dynamics [31]. In our case, the resonance condition ensures that $\xi_{1}-\theta_{L}-\theta=0$. 
Under this approximation, in the close vicinity of the resonance, the total Hamiltonian is given by

$$
H=p_{z}+\frac{M^{2}}{2 p_{z}}+\frac{\epsilon I}{\sqrt{p_{z}}}+a_{0} \exp \left(-\frac{\xi_{2}^{2}}{T^{2}}\right) \sqrt{\frac{\epsilon(I+L)}{2 p_{z}^{3 / 2}}} \sin \left[\xi_{1}-\theta_{L}-\theta\right],
$$

where $a_{0}=e A_{0} /\left(m c^{2}\right)$. It follows from the Hamiltonian (24) that

$$
-\frac{\partial H}{\partial \theta_{L}}=-\frac{\partial H}{\partial \theta}=-v_{p h} \frac{\partial H}{\partial z}=\dot{L}=\dot{I}=v_{p h} \dot{p}_{z}=\dot{w}
$$

where $w=\gamma+\epsilon^{2} r^{2} / 2$ is the total electron energy in the channel. The equation $\dot{w}=v_{p h} \dot{p}_{z}$ follows from the fact that $w+v_{p h} p_{z}=$ const is the integral of the motion defined by the initial Hamiltonian (4) (see also Ref. [20]).

Eq. (25) is the consequence of the energy-momentum conservation law for the resonant wave-particle interaction. It can be derived using the following simple reasoning. As a result of the interaction, an electron may absorb $n$ circularly-polarized photons. The energy gain in this case is $\Delta w=n \hbar \omega$, gain in longitudinal momentum is $\Delta p_{z}=n \hbar k_{z}=\Delta w / v_{p h}$ and the gain in angular momentum is $\Delta L=n \hbar$. Therefore $\Delta w / \Delta L=\dot{L} / \dot{\gamma}=\omega$. The transverse energy increment $\Delta w_{\perp}$ is related to the total energy change $\Delta w$ through $\Omega_{\beta} / \omega$. Since the betatron frequency is $\Omega_{\beta}=\Omega / \sqrt{p_{z}} \simeq \Omega / \sqrt{\gamma}$, the above relationship is consistent with Eq. (25).

For simplicity we will derive the Hamiltonian (24) with assumption $v_{p h}=$ $v_{g r}=c$. More general case $v_{p h} \neq v_{g r} \neq c$ is considered in Appendix. Waveparticle interaction destroys the constancy of the three actions $p_{z}, I$, and $L$. But, according to Eq. (25), the changes in these actions are identical because the interaction Hamiltonian depends on a single variable $\Psi=\xi_{1}-\theta_{L}-\theta$. Therefore, through an appropriate choice of the new action-angle variables, one can express the Hamiltonian as a function of a single action variable $p$ its conjugate angle variable $\Psi$. The canonical transformation is given by the generating function $S_{1}$ :

$$
S_{1}\left(p, z, C_{1}, \theta_{L}, C_{2}, \theta\right)=C_{1} \theta+\left(2 C_{2}-C_{1}\right) \theta_{L}+p\left(\theta_{L}+\theta-\xi_{1}\right) .
$$

The old $\left(p_{z}, I, L\right)$ and new $\left(p, C_{1}, C_{2}\right)$ actions are related according to

$$
p_{z}=p, I=C_{1}+p, L=2 C_{2}-C_{1}+p .
$$


The new actions $C_{1}$ and $C_{2}$ are the constants of motion. For example, an electron propagating straight along the channel with a vanishing transverse energy has (in the absence of the laser) $C_{1}=C_{2}=-p$.

Using inequalities $p_{z}^{2} \gg 1+\left(\mathbf{p}_{\perp}+e \mathbf{A}\right)^{2}, \epsilon \ll 1$ and $I>L$ we have the following inequalities for $p, C_{1}$ and $C_{2}: p^{3 / 2} / \epsilon>C_{1} \geq C_{2}$. It follow from our assumptions that $p=p_{z} \simeq \gamma$ and $\Delta p \simeq \Delta w$ if $v_{p h} \simeq c$.

In the new action-angle variables, the Hamiltonian (24) simplifies to

$$
H=\frac{M^{2}}{2 p}+\frac{\epsilon\left(p+C_{1}\right)}{\sqrt{p}}+a_{0} \exp \left(-\frac{t^{2}}{d^{2}}\right) \sqrt{\frac{\epsilon\left(p+C_{2}\right)}{p^{3 / 2}}} \sin \Psi,
$$

where $d=2 \lambda\left(M^{2} p^{-2}+2 \epsilon p^{-1 / 2}+2 \epsilon C_{1} p^{-3 / 2}\right)^{-1}=2 \lambda p^{2} /\left(M^{2}+\left\langle p_{\perp}^{2}\right\rangle\right)$ is the laser pulse duration in the coordinate system of the electron and $\lambda=T \omega$. Alternatively, $d$ is the time over which the laser pulse and the electron overlap.

The resonance condition is given by

$$
\dot{\Psi}=\frac{\partial H_{0}}{\partial p}=-\frac{M^{2}}{2 p^{2}}+\frac{\epsilon}{2 \sqrt{p}}-\frac{\epsilon C_{1}}{2 p^{3 / 2}}=0 .
$$

Hot electrons which interact with the laser pulse most strongly are characterized by the action variables $p$ and $C_{1}$ satisfying Eq. (29). From Eq. (27) and virial theorem we can obtain an identity

$$
\epsilon\left(p_{0}+C_{1}\right) \sqrt{p_{0}}=\epsilon I_{0} \sqrt{p_{0}}=p_{\perp, 0}^{2} / 2+\epsilon^{2} r_{0}^{2} / 2 \approx p_{\perp, 0}^{2},
$$

where $p_{\perp, 0}$ is the transverse momentum at the initial moment of time and $r_{0}$ is the initial radius of the electron location. This identity can be used to express $C_{1}$ and substitute it into the resonance condition (29). We now assume, for a moment, that an electron with the initial longitudinal energy $p_{0}$ and transverse momentum $p_{\perp, 0}$ is resonant with the laser immediately upon entering the channel, i. e. $p_{\text {res }}=p_{0}$ and $p_{\perp, \text { res }}=p_{\perp, 0}$, where the resonance energy is defined as in dimensional variables

$$
\gamma_{\text {res }}=p_{\text {res }} /(m c)=\left(\frac{M^{2} c^{2}+p_{\perp, 0}^{2}}{2 \epsilon m^{2} c^{2}}\right)^{2 / 3} .
$$

Using the fact that the betatron frequency is $\Omega_{\beta}=\Omega / \sqrt{\gamma}$ and expansion $\gamma m c^{2} \simeq p_{z} c+M^{2} c^{3} /\left(2 p_{z}\right)+p_{\perp, 0}^{2} c /\left(2 p_{z}\right)$ follows from Eq. (5), the condition (31) 
for betatron resonance can be rewritten in more familiar form [20]

$$
\Omega_{\beta}=\omega\left(1-\frac{v_{z}}{c}\right)
$$

In the limit $M=m$ and $p_{\perp, 0}=0$ the expression for resonant electron energy coincides with one calculated in in the limit $v_{p h}=c$. This expression is also similar to one known in the theory of ion channel laser [29, 32] in the theory of free electron lasers (FELs) [33, 34]. In FEL the electrons transversely oscillate in undulator instead of the betatron oscillation in ion channel. The wavelength of the radiation emitted in forward direction in FEL is given $[33,34]$

$$
\lambda=\frac{\lambda_{u}}{2 \gamma^{2}}\left(1+a_{w}^{2}\right)
$$

where $\lambda_{u}$ is the undulator wavelength and $a_{w}=e B_{0} \lambda_{u} /\left(2 \pi m c^{2}\right)$ is the undulator parameter. If we take into account that $p_{\perp}=m c a_{w}$ in FEL, $M=1$

and $\epsilon=\lambda_{u} /(\sqrt{\gamma} \lambda)=\Omega / \omega$ then we obtain Eq. (31).

\section{LASER ENERGY ABSORPTION AND MAGNETIC FIELD GENERATION}

In this Section we consider how the electron distribution function evolves under the influence of the laser pulse, resulting in the absorption of the laser energy and angular momentum. There are some indications from numerical simulations and experiments $[20,35]$ that some of electrons gain a little amount of the laser energy and some of the them gain a large one. Therefore two regimes can be envisioned: quasi-linear and strongly-nonlinear. In the quasi-linear regime, the energy change of the electron $w_{\sim}$ much smaller than its initial energy $w_{0}$. Another important feature of the quasi-linear regime is that the beam velocity always remains smaller than the group velocity of the laser pulse and the electrons quickly pass the pulse. Moreover, the laser pulse is assumed to be sufficiently short, so that the electron has enough time to slip through the pulse while remaining in the channel. This requires that $c T<\epsilon L_{c h} / \sqrt{p}$, where $L_{c h}$ is the length of the channel.

In the strongly-nonlinear regime, strong modification of the structure of electron orbits in phase space leads to the particle trapping and subsequent 
absorption of laser energy and angular momentum. As a result, the energy exchange between the electrons and the laser can be comparable or even exceed the initial electron energy. In this regime the beam velocity is close to the group velocity of the laser pulse and the electrons slowly pass the pulse so that the time of flight of an electron through the pulse is greater than the period of the nonlinear oscillation in the laser field:

$$
\frac{T v_{g r}}{\left|v_{z}-v_{g r}\right|}>t_{\text {trap }}
$$

where $t_{\text {trap }}$ is the characteristic period of trapped oscillations in the phase space.

\section{A. Quasi-linear regime}

In the quasi-linear regime, the energy absorbed by the hot electrons can be obtained using the perturbation theory. In analyzing this regime, we employ Madey's theorem [36], originally developed by John Madey for the analysis of FELs. Subsequently, Madey's theorem was generalized and applied to arbitrary Hamiltonian systems [37].

It follows from Eqs. (25), (27) the change in $w$ is proportional to the change in $p$ (Moreover in our case $v_{p h}=c \Delta w=\Delta p$ ). So to calculate change in electron energy we can calculate $\Delta p$. Formally, the last term in the Hamiltonian (28) is responsible for the wave-particle energy exchange. To zeroth order, there is no energy exchange:

$$
p^{(0)}=p_{0}=\text { const }, \quad \Psi^{(0)}\left(p_{0}, t, \Psi_{0}\right)=\frac{\partial H_{0}}{\partial p_{0}} t+\Psi_{0} .
$$

To first order, there is no overall energy exchange since equal numbers of electrons gain and lose energy. Individual particles gain/lose energy according to

$$
\Delta w^{(1)}=p_{\sim}=p^{(1)}-p_{0}=\int \frac{\partial H_{\sim}\left[p_{0}, \Psi^{(0)}\left(p_{0}, t, \Psi_{0}\right), t\right]}{\partial \Psi} d t,
$$

where $H_{\sim} \equiv H_{\text {int }}$ is the last term in the RHS of Eq. (28). Integrating Eq. (36) over $t$ yields

$$
p_{\sim}\left(p_{0}\right)=\sqrt{\pi} \epsilon a_{0} d \sin \Psi_{0} \sqrt{\frac{p_{0}+C_{2}}{p^{3 / 2}}} \exp \left[-\Delta \Psi\left(p_{0}\right)\right],
$$


where

$$
\Delta \Psi\left(p_{0}\right)=\frac{d^{2}}{4}\left(\frac{\partial H_{0}}{\partial p_{0}}\right)^{2}=\frac{\lambda^{2}}{4} \frac{\epsilon^{2}\left(C_{1}+\frac{M^{2}}{2 \epsilon \sqrt{p_{0}}}-p_{0}\right)^{2}}{\left(M^{2} p_{0}^{-1 / 2}+2 \epsilon p_{0}+2 \epsilon C_{1}\right)^{2}} .
$$

Not surprisingly, resonant electrons interact strongest with the laser because $\Delta \Psi$ vanishes at resonance; laser-particle energy exchange is exponentially diminished for non-resonant electrons. Because of the sinusoidal dependence of $p_{\sim}$ on the phase $\Psi_{0}$, the energy exchange $p_{\sim}\left(p_{0}\right)$ vanishes after averaging over the electron phases. The second order calculation is needed to find the change in $w$. One straightforward approach to deriving the phaseaveraged energy increment $\Delta w$ to second-order in the laser field $a_{0}$ is to use Madey's theorem [36]. According to this theorem, the second order change in $w$ is given by

$$
\Delta w=\Delta p=\left.\left\langle p^{(2)}-p_{0}\right\rangle\right|_{\Psi_{0}}=\left.\frac{1}{2} \frac{\partial}{\partial p_{0}}\left\langle p_{\sim}^{2}\left(p_{0}\right)\right\rangle\right|_{p_{1}=\text { const }, C_{2}=\text { const }} .
$$

Expressing $C_{1}$ and $C_{2}$ as function of $I_{0}$ and $L_{0}$ with help of Eq. (27), we can express the Madey's theorem (39) using more physically transparent actions $\mathbf{I}_{0}=\left(p_{0}, I_{0}, L_{0}\right)$ :

$$
\Delta p=\frac{1}{2} \widehat{G}\left\langle p_{\sim}^{2}\left(\mathbf{I}_{0}\right)\right\rangle,
$$

where

$$
\begin{aligned}
\left\langle p_{\sim}^{2}\left(\mathbf{I}_{0}\right)\right\rangle & =\left(I_{0}+L_{0}\right) D\left(p_{0}, I_{0}\right), \\
D\left(p_{0}, I_{0}\right) & =\frac{\pi \epsilon^{2} a_{0}^{2} \exp \left[-\Delta \Psi\left(p_{0}, I_{0}\right)\right]}{\left(M^{2} p_{0}^{-1 / 2}+2 \epsilon I_{0}\right)^{2}}, \\
\Delta \Psi\left(p_{0}, I_{0}\right) & =\frac{\lambda^{2}}{4} \frac{\epsilon^{2}\left(I_{0}+\frac{M^{2}}{\epsilon \sqrt{p_{0}}}-2 p_{0}\right)^{2}}{\left(M^{2} p_{0}^{-1 / 2}+2 \epsilon I_{0}\right)^{2}}, \\
\widehat{G} & \equiv\left(\frac{\partial}{\partial p_{0}}+\frac{\partial}{\partial I_{0}}+\frac{\partial}{\partial L_{0}}\right) .
\end{aligned}
$$

The absorbed energy per electron $Q$ can be calculated by averaging $\Delta p$ over the initial electron distribution function $F\left(\mathbf{I}_{0}\right)$,

$$
Q=\int \Delta p F\left(\mathbf{I}_{0}\right) d \mathbf{I}_{0}=-\frac{1}{2} \int\left\langle p_{\sim}^{2}\left(\mathbf{I}_{0}\right)\right\rangle \widehat{G} F\left(\mathbf{I}_{0}\right) d \mathbf{I}_{0} .
$$


The obtained expression is similar to the one for the rate of Landau damping [38]

$$
\Gamma_{L} \sim \int P_{\mathbf{k}}\left(\mathbf{k} \frac{\partial f}{\partial \mathbf{v}}\right)
$$

where $P_{\mathbf{k}}$ is the power of Cerenkov emission. In our case $\left\langle p_{\sim}^{2}\left(\mathbf{I}_{0}\right)\right\rangle$ is the power of "betatron" emission.

To calculate the absorbed energy, we consider a cold electron beam with the distribution function

$$
F\left(p_{z}, p_{\perp}, r\right)=\delta\left(p_{z}-p_{b}\right) \delta\left[H_{\perp}\left(p_{z}, p_{\perp}, r\right)-W_{\perp}\right],
$$

where $H_{\perp}=\epsilon I / \sqrt{p} \simeq p_{\perp}^{2} / p$ is the normalized transverse energy, and for simplicity we assume that $W_{\perp} \gg M / p$. Note that $W_{\perp}$ is proportional to the normalized electron emittance. The distribution function, expressed using the action variables, is given by

$$
F\left(\mathbf{I}_{0}\right)=\delta\left(p_{0}-p_{b}\right) \delta\left(\frac{\epsilon I_{0}}{\sqrt{p_{0}}}-W_{\perp}\right) \frac{\epsilon}{\sqrt{p_{0}}} .
$$

Assuming that electrons are uniformly distributed over the angular momentum $L$, integration of Eq. (42) over $p_{0}, I_{0}$ yields

$$
Q=\frac{\epsilon}{4 \sqrt{p_{b}} W_{\perp}} \int_{-\sqrt{p} W_{\perp} / \epsilon}^{\sqrt{p} W_{\perp} / \epsilon} \widehat{G}\left\langle p_{\sim}^{2}\left(p_{b}, \frac{\sqrt{p_{b}} W_{\perp}}{\epsilon}, L_{0}\right)\right\rangle d L_{0} .
$$

For long laser pulse $(\lambda=\omega T \gg 1)$

$$
\widehat{G}\left\langle p_{\sim}^{2}\left(\mathbf{I}_{0}\right)\right\rangle \simeq-\left.\left\langle p_{\sim}^{2}\left(\mathbf{I}_{0}\right)\right\rangle \widehat{G} \Delta \Psi\left(p_{0}, I_{0}\right)\right|_{p_{0}=p_{b}} .
$$

Then Eq. (46) takes the form

$$
Q \simeq-2 D\left(p_{b}, \frac{\sqrt{p_{b}} W_{\perp}}{\epsilon}\right) \widehat{G} \Delta \Psi\left(p_{b}, \frac{\sqrt{p_{b}} W_{\perp}}{\epsilon}\right) .
$$

Introducing $X=\epsilon \sqrt{p_{b}} / W_{\perp}$, we can rewrite Eq. (48) in the form

$$
\begin{aligned}
Q & \simeq \frac{\pi e^{2} A_{0}^{2}}{2 p_{b} c} \lambda^{2} X^{2}(1-2 X)(X-1) \exp [-\Delta \Psi(X, \lambda)], \\
\Delta \Psi(X, \lambda) & =\lambda^{2}(1-2 X)^{2} .
\end{aligned}
$$


The dependence of $Q$ on $X$ is shown on Fig. 2 and it is similar to one for small signal gain in FELs [34]. Spontaneous betatron emission is maximized for $X=1 / 2$. According to Eq. (31) and consistently with our earlier assumption of $W_{\perp} \gg M / p, X=1 / 2$ corresponds to the exact wave-particle resonance.

Note that the total energy gain (loss) by the electron ensemble vanishes for $X=1 / 2$. This phenomenon, which is the direct consequence of Madey's theorem, is well-know in the context of free-electron lasers: to achieve a small-signal gain, the electron energy $w$ has to be higher that the resonant energy $w_{\text {res }}$, calculated for given radiation frequency and magnetic undulator parameters [33]. The exact amount of the required for the peak gain energy excess $w_{\text {peak }}-w_{\text {res }}$ depends on the undulator length: the longer is the undulator, the smaller is the difference $w_{\text {peak }}-w_{\text {res }}$.

The total duration of the electron-laser interaction is limited by either the length of the plasma channel, or by the pulse duration (whichever is shorter). We assume that the laser pulse duration in the reference frame of the hot electron beam $d \ll \omega L / c$, where $L$ is the length of the channel. Assuming $\lambda \gg 1$, we find that $X \simeq 1 / 2+1 /(2 \sqrt{2} \lambda)$ maximizes the electron energy gain, while $X \simeq 1 / 2-1 /(2 \sqrt{2} \lambda)$ maximizes the small-signal gain of the laser pulse. The maximum of the absorbed energy as a function of the normalized pulse duration $\lambda=\omega T \gg 1$ is

$$
Q \simeq \frac{\pi e^{2} A_{0}^{2}}{16 p_{b} c} \frac{\lambda}{\sqrt{2}} \exp \left(-\frac{1}{2}\right) .
$$

Note that for the resonant electrons $\lambda \approx 2 \pi N_{\text {osc }}$, where $N_{\text {osc }}$ is the number of betatron oscillations executed by an electron while it stays inside the laser pulse.

Given the perturbed distribution function $F(p, I, L)$, the average magnetic field $\langle B\rangle$ can be computed according to Eq. (21):

$$
B_{\text {ind }}=\int\langle B(\mathbf{I})\rangle F(\mathbf{I}) d \mathbf{I}=\int \frac{2 e N \epsilon L}{I+\sqrt{I^{2}-L^{2}}} \frac{F(\mathbf{I})}{\sqrt{p}} d \mathbf{I} .
$$

The perturbed distribution function $F(p, I, L)$ can be obtained from the unperturbed distribution function $F\left(\mathbf{I}_{0}\right)$ by integration along the appropriate characteristics:

$$
\Delta p=\Delta I=\Delta L
$$


where Eq. (52) follows from Eq. (25). Instead of doing this, we use the fact that, initially, there is no magnetic field because electrons are uniformly distributed in $L$. Therefore, the induced quasi-static field can be calculated as

$$
\left.B_{\text {ind }}=\int\langle B(\mathbf{I})\rangle F(\mathbf{I}) d \mathbf{I}=\int\left\langle B\left[\mathbf{I}\left(\mathbf{I}_{0}\right)\right]\right)\right\rangle F\left(\mathbf{I}_{0}\right) d \mathbf{I}_{0},
$$

where $\mathbf{I}\left(\mathbf{I}_{0}\right)$ is the set of perturbed action variables of the electron which starts out with the set of unperturbed action variables $\mathbf{I}_{0}$.

In the quasi-linear absorption regime, $B\left[\mathbf{I}\left(\mathbf{I}_{0}\right)\right]$ can be Taylor expanded:

$$
B\left[\mathbf{I}\left(\mathbf{I}_{0}\right)\right]=B\left(\mathbf{I}_{0}\right)+\Delta p \widehat{G} B\left(\mathbf{I}_{0}\right)+\frac{\left\langle p_{\sim}^{2}\right\rangle}{2} \widehat{G}^{2} B\left(\mathbf{I}_{0}\right),
$$

where we performed the averaging over $\Psi_{0}$. Using generalized Madey's theorem (39), we can reduce Eq. (54) to a more convenient form

$$
B\left[\mathbf{I}\left(\mathbf{I}_{0}\right)\right]=B\left(\mathbf{I}_{0}\right)+\frac{1}{2} \widehat{G}\left[\left\langle p_{\sim}^{2}\left(\mathbf{I}_{0}\right)\right\rangle \widehat{G} B\left(\mathbf{I}_{0}\right)\right] .
$$

Integrating Eq. (53) by parts yields

$$
B_{\text {ind }}=-\int \frac{\left\langle p_{\sim}^{2}\left(\mathbf{I}_{0}\right)\right\rangle \widehat{G} B\left(\mathbf{I}_{0}\right)}{2} \widehat{G} F\left(\mathbf{I}_{0}\right) d \mathbf{I}_{0}
$$

Equation (56) for the magnetic field bears some similarity with Eq. (42) for the absorbed energy, except for an additional factor $\widehat{G} B\left(\mathbf{I}_{0}\right)$ :

$$
\widehat{G} B\left(\mathbf{I}_{0}\right)=\frac{2 e N}{I_{0}+\sqrt{I_{0}^{2}-L_{0}^{2}}} \frac{\epsilon}{\sqrt{p_{0}}}\left(-\frac{L_{0}}{2 p_{0}}+\sqrt{\frac{I_{0}-L_{0}}{I_{0}+L_{0}}}\right) .
$$

The field strength of the generated $B$-field is calculated by integrating Eq. (56) by parts and assuming an electron distribution function given by Eq. (45):

$$
B_{\text {ind }}=\int \frac{F\left(\mathbf{I}_{0}\right)}{2} \widehat{G}\left[\left\langle p_{\sim}^{2}\left(\mathbf{I}_{0}\right)\right\rangle \widehat{G} B\left(\mathbf{I}_{0}\right)\right] d \mathbf{I}_{0} .
$$

After straightforward but cumbersome algebra, we obtain

$$
B_{i n d} \simeq\left(1-\frac{\pi}{4}\right)(2-X) \frac{2 e N \Omega}{c} \frac{\Omega}{\omega} \frac{m c^{2}}{W_{\perp}} \frac{Q}{p_{b} c},
$$


where $Q$ is the absorbed energy per electron given by Eq. (49).

Introducing the average electron beam density $n_{b}$ in the channel according to

$$
n_{b} \simeq \frac{N}{\pi r_{\max }^{2}} \simeq \frac{1}{2 \pi} \frac{N \omega \Omega}{c^{2}} \sqrt{\frac{p_{z}}{m c}} \frac{m c^{2}}{\omega I_{0}} \simeq \frac{1}{2 \pi} \frac{N \Omega^{2}}{c^{2}} \frac{m c^{2}}{W_{\perp}},
$$

Eq. (59) can be recast in a more intuitive form

$$
\frac{\omega_{c e}}{\omega} \simeq\left(1-\frac{\pi}{4}\right)(2-X) \frac{\omega_{b e}^{2}}{\omega^{2}}\left(\frac{Q}{p_{b} c}\right),
$$

where $\omega_{b e}^{2}=4 \pi e^{2} n_{b} / m$, and $\omega_{c e}=e B_{i n d} / m c$ is the nonrelativistic electron cyclotron frequency. Assuming that $\omega_{b e}^{2} \simeq \alpha \Omega^{2}$, where $\alpha=n_{e} / n_{i}$ is the degree of electron evacuation in the channel, we rewrite Eq. (61) as

$$
\frac{\omega_{c e}}{\omega} \simeq\left(1-\frac{\pi}{4}\right)(2-X) \alpha \epsilon^{2}\left(\frac{Q}{\gamma_{b} m c^{2}}\right)
$$

where relativistic gamma-factor of the electron, $\gamma_{b}$, is used instead of $p_{b}$ because in approximation $\gamma \simeq p=p_{z}^{2} \gg 1+\left(\mathbf{p}_{\perp}+e \mathbf{A}\right)^{2}$. Note that $\gamma_{b} m c^{2}$ in Eq. (62) is the initial electron energy. Equation (62) loses validity when $Q_{b}$ becomes comparable to $\gamma_{b} m c^{2}$. Therefore, the largest reliable value of the magnetic field which can be deduced from the quasi-linear theory is of the order of $B_{\text {ind }} / B_{\omega} \sim \epsilon^{2}$, where $B_{\omega}=m c \omega / e$. For a $1 \mu \mathrm{m}$ laser pulse $B_{\omega} \approx$ $100 \mathrm{MG}$. Therefore, for a tenuous plasma with density $2 \times 10^{19} \mathrm{~cm}^{-3}$ (which corresponds to the plasma density measured in the RAL experiment [15]), the predicted magnetic field is at most $0.5 \mathrm{MG}$. Since this prediction is almost an order of magnitude below the recently measured magnetic field strength [15], and since the energy transferred to the electrons can be much higher than their initial energy [21], the strongly nonlinear calculation is well motivated.

\section{B. Strongly nonlinear regime}

The perturbative treatment of the previous section is not applicable in the strongly nonlinear regime, where the electron energy change can be very significant, and electrons can even become trapped in the laser field. Under condition (34), the variation of the laser wave amplitude, seen by an electron executing a nonlinear oscillation, is small. Therefore, adiabatic theory is adequate for describing the electron motion. 
The adiabatic invariant of the electron motion can still be broken if the electron orbit in the phase space crosses a separatrix at a particular value of the system parameter [39, 40, 41, 42, 43, 44]. At this moment the electron becomes trapped by the wave, or conversely becomes free after being trapped. The breaking of the adiabatic invariant leads to the absorption of the wave energy. Incidentally, the Hamiltonian (28) is somewhat similar to the one describing the nonlinear electron motion in a strong plasma wave [39, 40, 41], or close to the cyclotron resonance $[42,43,44]$. Below, we demonstrate that the crossing separatrix by electron also occurs in our system.

Assuming that the absorbed energy in this regime is much larger than initial energy, we neglect the $C_{2}$ in the Hamiltonian given by Eq. (28), and consider a simplified Hamiltonian

$$
H \simeq \frac{M^{2}}{2 p}+\frac{\epsilon\left(p+C_{1}\right)}{\sqrt{p}}+a(t) \sqrt{\epsilon} p^{-1 / 4} \cos \Psi,
$$

where $a(t)=a_{0} \exp \left(-t^{2} / d^{2}\right)$. It follows from Eq. (27) that condition $C_{2}=0$ implies that $I>L>0$. The equations of motion in the $(p ; \Psi)$ plane are given by

$$
\begin{aligned}
\frac{d p}{d t} & =a(t) \sqrt{\epsilon} p^{-1 / 4} \sin \Psi \\
\frac{d \Psi}{d t} & =\left(-\frac{M^{2}}{2 p^{2}}+\frac{\epsilon}{2 \sqrt{p}}-\frac{\epsilon C_{1}}{2 p^{3 / 2}}\right)-\frac{a(t) \sqrt{\epsilon}}{4 p^{5 / 4}} \cos \Psi
\end{aligned}
$$

The bracketed term in the RHS of Eq. (65) is equal to the detuning from the betatron resonance. It vanishes when $p=p_{\text {res }}$, where $p_{\text {res }}$ is defined by Eq. (29). When $p<p_{\text {res }}$, the bracketed term is negative. The $\Psi$-dependent term in the RHS of Eq. (65) is the so called inertial bunching, well known in the theory of the Cyclotron Autoresonant Maser (CARM) [45, 47, 46]. In the context of the ion-channel laser, this term was identified by Whittum $[29,32]$ as the "debunching" term.

A somewhat similar system of equations was previously derived by Tsakiris et. al. [28], and used for calculating the electron energy gain. Linearly polarized laser pulse was assumed in Ref. [28]. Our calculation differs from Ref. [28] in two respects. First, it assumes a circularly polarized laser pulse since the focus of our calculation is the magnetic field generation via the IFE. 
Second, by preserving the Hamiltonian nature of the equations of motion, we retained the inertial bunching term which was omitted in Ref. [28].

There are several fixed points in the phase space: an elliptic fixed point at $\Psi=\pi$, and a pair of fixed (elliptic and hyperbolic) points at $\Psi=0$ (see Fig. 2). Contrary to the nonlinear electron dynamics near the cyclotron resonance $[42,43,44]$ in our case no bifurcation of phase space happens and all fixed points exist at an arbitrary value of $a$ like in the problem of electron trapping in plasma wave [39, 40,41]. For constant laser amplitude $a$, the Hamiltonian is a constant of motion, and the electron orbits are given by the $H=$ const curves in the $(p ; \Psi)$ phase space.

A particle passing through the laser pulse experiences the slowly changing with time laser amplitude. The Hamiltonian is then no longer a constant of motion. Under the adiabatic condition (34), electron transitions from one curve to another are governed by the conservation of the adiabatic invariant

$$
J=\oint p d \Psi
$$

where the integral is taken over one complete period of motion in phase space. If electron is not trapped, $J$ is the area under a curve of constant $H$; for trapped electrons $J$ is the area enclosed by one of the closed contours surrounding the elliptic fixed point. In the limit $a \longrightarrow 0, J$ is proportional to the electron energy. Therefore, the conservation of $J$ must be broken for an electron in order to gain energy as it passes through separatrix (i. e. through the laser pulse.)

The mechanism of the electron heating in the nonlinear regime is schematically demonstrated by Fig. 3, where we present sequence of phase space snapshots of the dynamical system governed by the Hamiltonian 28 with parameters $p_{0}=3.9, p_{\perp, 0}=2, C_{2}=0$, and $\epsilon=0.1$. The relation between $p_{\perp, 0}$ and $C_{1}$ is $p_{\perp, 0}^{2}=\epsilon\left(C_{1}+p_{0}\right) / \sqrt{p_{0}}$ and follows from Eqs. (27) and (30). A similar sequence of phase space snapshots (albeit for a very different physical system) was produced by Nevins et. al. [42] to illustrate the nonlinear regime of the cyclotron heating. The initial particle distribution of cold electron beam is shown as a thick line in Fig. 3(a). For simplicity, we assumed fast electrons $\left(p \gg p_{\perp}, 1+a^{2}\right)$ which are still moving slower than laser pulse $\left(v_{z}<v_{g r}\right)$ (see Fig. 4). Such electrons can be, for example, pre-accelerated by the plasma wave excited in the front of the laser pulse. If the pre-acceleration takes place where the amplitude of the laser pulse is relatively small, then 
the electrons are not initially trapped. Another plausible scenario (which is outside of the scope of this work but can be described in our model) can be envisioned: electrons can be accelerated by the plasma wave behind the laser pulse and gain significant energy to overtake the pulse. An additional (and the most significant) energy gain will then occur during the electron passage through the pulse.

As the electrons move into the center of the laser pulse, the field strength parameter $a$ increases, and the region of trapped orbits about the elliptic fixed point expands. As the trapped region expands, the phase volume flows from the region of open orbits below the separatrix, through the hyperbolic fixed points, and into the trapped region. If the wave amplitude is sufficiently large, all of the phase volume below separatrix is pulled into the trapped region [see Fig. 3(b)]. For the present simulation parameters, trapping occurs for $a_{t r}=0.7$. Below we derive a qualitative criterion for the onset of trapping.

As electrons move out of the pulse, $a$ decreases and the trapped region collapses. The phase volume is expelled through the hyperbolic fixed point into both the regions above and below the separatrix. Therefore, electrons are pushed back into the passing region, but roughly half of them are expelled above the separatrix $[39,42,44]$. Those electrons that are pulled out into the trapped region from below the separatrix and end up above the separatrix gain energy and angular momentum.

Since $p \simeq \gamma$ in our model we will use the kinetic energy of the electron, $\gamma$ instead of the longitudinal momentum of the electron, $p$. In Figs. 5(a),(b) we show a typical trajectory of an electron which crossed the separatrix twice without the net energy gain. Our numerical simulation confirms that the fraction of such electrons is about 1/2. A phase-space trajectory of another electron, which also crossed the separatrix twice, but absorbed some net energy, is shown in Figs. 5(c),(d). Numerical simulation indicates that approximately half of the electrons do not change energy and return to $\gamma_{0}$, another half of the electrons reach the final energy $\gamma_{f}$, and a small fraction of the electrons have the final value of energy between $\gamma_{0}$ and $\gamma_{f}$. The last portion appears because the adiabaticity condition is not strictly fulfilled $\left[\left|v_{z}-v_{g r}\right| t_{\text {trap }} /\left(T v_{g r}\right) \neq 0\right]$.

To estimate the energy gain in nonlinear regime, we should use the fact that adiabatic invariant $J$ is conserved before the separatrix crossing. Unfortunately the Hamiltonian (63) is too complex to provide analytical calculation of $J$ and we will use the fact (following from numerical simulations) that 
the Hamiltonian does not change essentially during interaction (see Fig. 6). Before electron enters the pulse $(a \simeq 0)$, its Hamiltonian is equal to

$$
H(t=-\infty)=H_{0}=\frac{1}{2 \gamma_{0}}+\frac{\epsilon\left(\gamma_{0}+C_{1}\right)}{\sqrt{\gamma_{0}}} \simeq \frac{1+p_{\perp, 0}^{2}}{2 \gamma_{0}}
$$

After electron passing the laser pulse $a$ is again equal to 0 . The equation $H(t=+\infty)=H(p, a=0) \simeq H_{0}$ has two solutions, $\gamma_{0}$ and $\gamma_{f}$, where

$$
\gamma_{f} \simeq \frac{p_{\perp, 0}^{4}}{\epsilon^{2} \gamma_{0}^{2}}
$$

To derive this expression we assume that $p_{\perp, 0}^{2} \gg 1$ and use the fact that $\gamma_{f} \gg \gamma_{0}$. Note that, contrary to the quasi - linear regime of the energy absorption when $\Delta p=\Delta w$, in strongly nonlinear regime $\gamma_{f} \gg \gamma_{0}$ and $\Delta p=$ $\Delta w \simeq \gamma$. For the parameters of the numerical simulations presented in Figs. 5,6 $\left(\gamma_{0}=5.9, p_{\perp, 0}=2, \epsilon=0.12\right)$, Eq. (68) predicts $\gamma_{f} \simeq 25$, in fairly good agreement with the numerical result $\gamma_{f} \simeq 24$. Hence, the averaged energy gain is

$$
\langle\Delta \gamma\rangle=\frac{\gamma_{f}+\gamma_{0}}{2} \simeq \frac{p_{\perp, 0}^{4}}{2 \epsilon^{2} \gamma_{0}^{2}} .
$$

Electron trapping occurs when the initially injected particles cross the separatrix. Using the conservation of the adiabatic invariant $J(H, a)=$ $J\left(H_{0}, 0\right)=$ const we can express $H$ as function of $a: H=H\left(H_{0}, a\right)$. This function is not valid at the moment when the particle orbit cross the separatrix in the phase space and the adiabatic invariant becomes broken. Separatrix is a characterized by the certain value of the Hamiltonian $H_{s e p}$. Separatrix crossing occurs at $a=a_{t r}$ when the given electron orbit starting with parameters $H_{0}$ and $a=0$ at $t=-\infty$ in the phase space becomes the separatrix. The value of $a_{t r}$, at which the separatrix crossing (or particle trapping) occurs, can be estimated from the equation $H\left(H_{0}, a=a_{t r}\right) \simeq H_{\text {sep }}$. Electrons are trapped in the wave if the peak laser amplitude $a_{0}$ satisfies

$$
a_{0}>a_{t r}\left(\gamma_{0}, p_{\perp, 0}, a_{0}\right) .
$$

The range of parameters $\gamma_{0}, p_{\perp, 0}^{2}, a_{0}$ for which electrons can be accelerated in the strongly nonlinear regime can be obtained from the

$$
\gamma_{f}\left(\gamma_{0}, p_{\perp, 0}\right)>\gamma_{0}
$$


and Eq. (70).

Using expression (69) for mean energy gain, the absorbed energy can be obtained for electrons with distribution function $F\left(\gamma_{0}, p_{\perp, 0}\right)$

$$
Q=\iint_{S_{r e s}} d \gamma_{0} d p_{\perp, 0} \Delta \gamma\left(\gamma_{0}, p_{\perp, 0}\right) F\left(\gamma_{0}, p_{\perp, 0}\right),
$$

where $S_{\text {res }}$ is the domain of $\gamma_{0}, p_{\perp, 0}$ at which strongly nonlinear acceleration regime occurs. For cold electron beam with distribution function (44), where $p_{b}$ and $W_{\perp}$ belong to the resonance domain $S_{r e s}$, we obtain

$$
Q \simeq \frac{W_{\perp}^{2}}{2 \epsilon^{2}}=W_{\perp} \frac{W_{\perp}}{m c^{2}} \frac{\omega^{2}}{2 \Omega^{2}} .
$$

In the nonlinear absorption regime, electrons gain large amounts of energy and angular momentum after interaction with laser pulse $\left(\Delta \gamma>\gamma_{0} \simeq p_{b}\right.$, $\Delta I>I_{0}$ and $\left.\Delta L>L_{0}\right)$. So in strongly nonlinear regime all resonant electrons after interaction with laser pulse have $I \simeq L \simeq \Delta I$ and perform circular motion with $r=\sqrt{\Delta I /(\epsilon \sqrt{\Delta \gamma})}$. Then, the generated magnetic field in this regime is

$$
B_{\text {ind }} \simeq 2 e N \epsilon \int_{S_{\text {res }}} \frac{F_{0}\left(\mathbf{I}_{0}\right)}{\sqrt{\Delta \gamma\left(\mathbf{I}_{0}\right)}} d \mathbf{I}_{0},
$$

where integration in this expression is taken over the resonance particle $S_{\text {res }}$ Eq. (70).

Using the electron distribution function given by Eq. (44) yields

$$
B_{\text {ind }} \simeq \frac{2 \sqrt{2} e N \epsilon^{2}}{W_{\perp}}=\frac{2 e N \epsilon}{\sqrt{Q}}=\frac{2 e N \Omega}{c} \frac{m c^{2}}{\sqrt{Q}} .
$$

Since $I_{f} \simeq \gamma_{f}$ we can present Eq. (60) in the form

$$
\left\langle n_{b}\right\rangle \simeq \int \frac{N}{\pi r_{\max }^{2}} F_{0}\left(\mathbf{I}_{0}\right) d \mathbf{I}_{0} \simeq \frac{N \epsilon}{2 \pi \sqrt{Q}}=\frac{N \omega \Omega}{c^{2}} \sqrt{\frac{m c^{2}}{Q}} .
$$

Thus, the axial magnetic field generated in the strongly nonlinear regime can be rewritten in the form

$$
\frac{\omega_{c e}}{\omega} \simeq \frac{\left\langle\omega_{b e}^{2}\right\rangle}{\omega^{2}} \simeq \alpha \epsilon^{2}
$$


Equation (77), derived for the strongly nonlinear regime, yields no surprises or new results beyond what one could expect from pushing Eq. (62) to its applicability limit. For the parameters of the recent experiment at RAL [15], the peak magnetic field can be estimated as less than 1 MG. This estimate is almost three times smaller that the experimentally measured field.

Interestingly, Eq. (77) indicates that the magnetic field does not depend on the actual amount of energy (or angular momentum) gained by the electrons. This result can be understood as follows: Since magnetic field is determined by the azimuthal electron current $j_{\phi}$, and $j_{\phi}=-e n_{b} v_{\phi}$, what ultimately matters for B-field generation is the azimuthal electron velocity $v_{\phi}$. But the increase $\Delta v_{\phi}=\Delta p_{\phi} / p$, where $\Delta p_{\phi}$ is the electron momentum increment. Therefore, as electrons gain angular momentum, they also gain energy, increasing $p$. Thus, the angular momentum increase is offset by the relativistic increase of the electron mass. This mass increase is, ultimately, responsible for the weakness of the magnetic field predicted by Eq. (77).

To explain the experimental result, one may have to include collective effects which could lead to the angular momentum re-distribution between the highly relativistic and non-relativistic electrons. Also, fast electrons produced by the laser trapping can excite plasma waves. These plasma waves, supported by the ambient plasma (in and outside of the ion channel), can significantly slow down fast electrons. The longitudinal electric field of the plasma wave does not change the angular momentum of the electrons. Its role is to remove a significant fraction of the total energy imparted by the laser. As a result, fast particles can absorb significant amount of energy (and angular momentum) from the laser without becoming very heavy. For example, to explain the experimentally observed magnetic field 2.5 MG requires that hot electrons loose about $2 / 3$ of the energy gained from the laser to plasma wave generation. Also numerical simulations show [48] that the highest electron density is outside the channel an near the channel border since the electric field of the channel ions attracts the electrons outside the channel too. The number of these electrons substantially exceeds the electrons in the channel. These electron can also perform circular motion around the channel and generate ultrahigh intensity magnetic field. 


\section{DISCUSSION AND CONCLUSION}

In this paper we have analyzed the dynamics of the wave-particle interaction in an ion channel, with the emphasis on the magnetic field generation, or IFE. For the first time, to our knowledge, a simple and intuitive physical picture of the axial magnetic field generation through the angular momentum transfer from the laser pulse to the hot electrons is developed. Since the angular momentum absorption requires the resonant photon absorption, we identified one possible mechanism as the betatron resonance between the hot electrons in the partially-evacuated ion channel and the co-propagating laser pulse. This resonance has been recently identified [20, 21, 28] as the cause of the significant electron energy gain directly from the laser pulse. Here, we point out that if the laser pulse is circularly polarized, then another by product of such a resonant wave-particle interaction is the axial magnetic field generation (also known as IFE).

We calculated the magnitude of the magnetic field in two regimes: quasilinear and strongly nonlinear. The quasi-linear regime requires the presence of the large population of hot resonant electrons, while the strongly nonlinear regime is valid for a broad range of electron energies. The generalized Madey's theorem was used to calculate the electron energy gain and magnetic field generation in the quasi-linear regime. In the strongly nonlinear regime, we related the net electron energy gain to the structural transition of the resonance electron orbits in the phase space as result of the separatrix crossing during the adiabatic change of the laser field strength. For both regimes the absorbed angular momentum is proportional to the absorbed energy that is the manifestation of the resonance nature of the radiation energy absorption phenomenon [49].

While calculations were performed for the circularly polarized laser pulse, all the results pertaining to the energy transfer are also valid for the linearly polarized wave. In this regard, our work extends the earlier simulations [20, 21] and theoretical calculations [28] which predicted a significant electron energy gain. By using the Hamiltonian approach from the start, we were able

to retain some of the physics [e. g., force bunching term in Eq. (65)] which was neglected in Ref. [28]. Use of the adiabatic approximation also enabled us to treat the strong electron acceleration observed in these simulations when the electrons overtake the laser pulse since all phenomena like electron trapping in laser wave and separatrix crossing in the phase space occur in 
this case too.

One of the motivations for this work was to interpret the recent experimental measurements [15] of the IFE during relativistic laser-plasma interactions. While we were able to explain the effect qualitatively as being due

to the resonant absorption of the laser angular momentum by the plasma electrons, our estimates of the magnetic field strength are lower than the experimentally measured. We speculate that this discrepancy is due to the fact that the model used in this paper is not fully self-consistent. For example, it neglects the laser depletion by the hot electrons. All interactions of the hot electrons with the background plasma are also neglected. Such interactions include, for example, acceleration or deceleration of the hot electrons by the plasma waves supported by the background plasma.

Our theory would have predicted a much larger magnetic field if hot electrons were continuously slowed down by their interaction with the surrounding plasma. For example, one could envision the energy loss associated with the generation of the plasma waves by the fast electrons. Including this effect would have brought the estimates into better agreement with the experimental data. Moreover, to estimate the energy gain and magnetic field strength, we used a very simple distribution function. Further investigations will include the more realistic electron distribution functions, as well as the more self-consistent analysis of the interaction between fast electrons and the ambient plasma.

\section{ACKNOWLEDGMENTS}

One of the authors (I. K.) gratefully acknowledges the hospitality of Princeton Plasma Physics Laboratory. This work was support in part by US DOE Division of High Energy Physics, by the Russian Fund for Fundamental Research (Grants No 01-02-16575, No 01-02-06488), by INTAS (Grant YSF 00-46) and by RAS (Grant N 1999-37). 


\section{APPENDIX: BETATRON RESONANCE IN THE CASE $v_{p h} \neq c$}

In general case $p_{p h}^{2} \neq \infty$ the Hamiltonian (28) have an additional term

$$
H=-\frac{p}{2 p_{p h}^{2}}+\frac{M^{2}}{2 p}+\frac{\epsilon\left(p+C_{1}\right)}{\sqrt{p}}+A_{0} \exp \left(-\frac{t^{2}}{d^{2}}\right) \sqrt{\frac{\epsilon\left(p+C_{2}\right)}{p^{3 / 2}}} \sin \Psi,
$$

where $d=2 \lambda\left(M^{2} p^{-2}-p_{p h}^{-2}+2 \epsilon p^{-1 / 2}+2 \epsilon C_{1} p^{-3 / 2}\right)^{-1}$.

The resonance condition is

$$
\dot{\Psi}=\frac{\partial H_{0}}{\partial p}=-\frac{1}{2 p_{p h}^{2}}-\frac{M^{2}}{2 p^{2}}+\frac{\epsilon}{2 \sqrt{p}}-\frac{\epsilon C_{1}}{2 p^{3 / 2}}=0 .
$$

The resonance condition can be rewritten as follows

$$
\frac{\partial H_{0}}{\partial p}=\frac{1}{2 p^{3 / 2}}\left(\frac{\sqrt{p}}{2 p_{p h}^{2}}-\epsilon\right)\left(C_{1}+\frac{M^{2}}{\epsilon \sqrt{p}}-p\right)-R,
$$

where $2 R=p_{p h}^{-2}+C_{1} p^{-1}+M^{2} p^{-3 / 2} \epsilon^{-1}$. To derive the condition when we can neglect term $R$ in Eq. () we introduce the effective neutralization factor in the channel, $f=n_{e} / n_{i}$, then $\omega_{b e}^{2} \simeq f \epsilon^{2}$ and $p_{p h}^{-2} \simeq f \epsilon^{2}$. Then $R$ can be neglected if $\left\{p, \epsilon^{-1}\right\} \gg\left|\mathbf{p}_{\perp}+\mathbf{A}\right|$. At the resonance $p_{\text {res }}=p_{0}$ and $p_{\perp, \text { res }}=p_{\perp, 0}$ and the resonance energy is

$$
\begin{aligned}
\gamma_{\text {ras }} & \simeq p_{\text {res }}=4 \epsilon^{2} p_{p h}^{4}, \\
\gamma_{\text {res }} \simeq p_{\text {res }} & =\left(\frac{M^{2}+p_{\perp, 0}^{2}}{2 \epsilon}\right)^{2 / 3} .
\end{aligned}
$$

In the limit $M=1$ and $p_{\perp, 0}=0$ the expression for resonance electron energy coincides with ones calculated in [20] (see Fig. 4 therein).

\section{References}

[1] A. Modena, A. E. Dangor, Z. Najmudin, C. E. Clayton, K. Marsh, C. Joshi, V. Malka, C. B. Darrow, C. Danson, D. Neely, and F. N. Walsh, Nature 377, 606 (1995). 
[2] A. Ting, C. I. Moore, K. Krushelnick, C. Manka, E. Esarey, P. Sprangle, R. Hubbard, H. R. Burris, R. Fischer, and M. Baine, Phys. Plasmas 4, 1889 (1997).

[3] R. Wagner, S.-Y. Chen, A. Maksimchuk, and D. Umstadter, Phys. Rev. Lett. 78, 3125 (1997).

[4] K. Krushelnick, E. L. Clark, Z. Najmudin, M. Salvati, M. I. K. Santala, M. Tatarakis, A. E. Dangor, V. Malka, D. Neely, R. Allott, and C. Danson, Phys. Rev. Lett. 83, 737 (1999).

[5] A. Maksimchuk, S. Gu, K. Flippo, D. Umstadter, and V. Yu. Bychenkov, Phys. Rev. Lett. 84, 4108 (2000).

[6] M. Borghesi, A. J. MacKinnon, A. R. Bell, R. Gailard, and O. Willi, Phys. Rev. Lett. 81, 112 (1998).

[7] J. A. Stamper, Laser and Part. Beams, 9, 841 (1991).

[8] R. N. Sudan, Phys. Rev. Lett. 20, 3075 (1993).

[9] Y. Horovitz, S. Eliezer, A. Ludmirsky, Z. Henis, E. Moshe, R. Spitalnik, and b. Arad, "Measurements of Inverse Faraday Effect and Absorption of Circularly Polarized Laser Light in Plasmas", Phys. Rev. Lett. 78, 1707 (1997).

[10] S. C. Wilks, W. L. Kruer, M. Tabak, and A. B. Langdon, Phys. Rev. Lett. 69, 1383 (1992); R. J. Mason and M. Tabak, Phys. Rev. Lett. 80, 524 (1998).

[11] L. M. Gorbunov, P. Mora, and T. M. Antonsen, Phys. Plasmas 4, 4358 (1997).

[12] L. P. Pitaevskii, Sov. Phys. JETP 12, 1008 (1961).

[13] A. D. Steiger and C. H. Woods, Phys. Rev. A 5, 1467 (1972).

[14] T. Lehner, Europhys. Lett. 50, 480 (2000).

[15] Z. Najmudin, M. Tatarakis, A. Pukhov, E. L. Clark, R. J. Clarke, A. E. Dangor, J. Faure, V. Malka, D. Neely, M. I. K. Santala, and K. Krushelnick, Phys. Rev. Lett. 87, 215004 (2001). 
[16] A. Sh. Abdullaev and A. A. Frolov, Sov. Phys. JETP 54, 493 (1981).

[17] Z. H. Sheng and J. Meyer-ter-Vehn, Phys. Rev. E 54, 1833 (1996).

[18] L. M. Gorbunov, P. Mora and T. M. Antonsen, Jr., Phys. Rev. Lett. 76, 2495 (1996).

[19] V. I. Berezhiani, S. M. Mahajan and N. L. Shatashvili, Phys. Rev. E 55, 995 (1997).

[20] A. Pukhov, Z. M. Sheng and J. Meyer-ter-Vehn, Phys. Plasmas, 6, 2847 (1999).

[21] C. Gahn, G. Tsakiris, A. Pukhov, et al., Phys. Rev. Lett. 83, 4772 (1999).

[22] I. Yu. Kostyukov, G. Shvets, N. J. Fisch, J. M. Rax, Laser Part. Beams 19, 133 (2001); ibid, Bull. Am. Phys. Soc. 47, 39 (2000)

[23] M. H. Key, M. D. Cable, T. E. Cowan, et. al. Phys. Plasmas, 5, 1966 (1998).

[24] K. B. Wharton, C. Brown, B. A. Hammel, S. Hatchett, M. H. Key, et al., Phys. Rev. Lett. 81, 822 (1998).

[25] A. Pukhov and J. Meyer-ter-Vehn, Phys. Rev. Lett. 79, 2686 (1997).

[26] A. Pukhov and J. Meyer-ter-Vehn, Phys. Rev. Lett. 76, 3975 (1997).

[27] A. Pukhov and J. Meyer-ter-Vehn, Phys. Plasmas, 5, 1880 (1998).

[28] G. D. Tsakiris, C. Gahn, V. K. Tripathi, Phys. Plasmas, 7, 3017 (2000).

[29] D. H. Whittum, Theory of the Ion-Channel Laser, Ph. D. Thesis, September 1990 (unpublished).

[30] R. C. Davidson, Physics of Nonneutral PLasmas (Addison-Wesley, Redwood City, California, 1990).

[31] A. J. Lichtenberg and M. A. Lieberman, Regular and Stochastic Motion (Springer-Verlag, New York, 1983). 
[32] D. H. Whittum, A. M. Sessler, and J. M. Dawson, Phys. Rev. Lett. 64, $2511(1990)$.

[33] W. B. Colson, J. C. Gallardo, and P. M. Bosco, Phys. Rev. A 34, 4875 (1986).

[34] G. Dattoli, L. Giannessi, A. Renieri, A. Torre, in Progress in Optics XXXI, ed. by E. Wolf, p. 321 (Elsevier Science Publishers B.P., London, 1993).

[35] V. Malka, J. Faure, J. P. Marques et al., Phys. Plasmas 8, 2605 (2001)

[36] J. M. J. Madey, Nuovo Cimento B 50, 64 (1979).

[37] G. M. Fraiman and I. Yu. Kostyukov, Phys. Plasmas 2, 923 (1995).

[38] V. V. Zhelezniakov, Radiation in Astrophysical Plasmas (Astrophysics and Space Science Library), V. 204 (Kluwer Academic Pub, Boston, 1996).

[39] R. Aamodt, E. Jaeger, Phys. Fluids, 17, 1386 (1974).

[40] R. Best, Physics, 40, 182 (1968).

[41] A. S. Bakai and Yu. P. Stepanovskii, Adiabatic Invariants (Naukova Dumka, Kiev, 1981)

[42] W. M. Nevins, T. D. Rognlien and B. J. Cohen, Phys. Rev. Lett. 59, 60 (1987).

[43] E. V. Suvorov and M. D. Tokman, Fiz. Plazmy, 14, 950 (1988).

[44] I. A. Kotel'nikov and G. V. Stupakov, Phys. Fluids B, 2, 882 (1990).

[45] K. R. Chu and J. L. Hirshfield, Phys. Fluids, 21, 461 (1978).

[46] C. Chen and J. S. Wurtele, Phys. Rev. A 40, 489 (1989).

[47] K. R. Chen, J. M. Dawson, A. T. Lin, and T. Katsouleas, Phys. Fluids B 3, 1270 (1990). 
[48] M. Borghesi, A. J. Macckinnon, L. Barringer, et al., Phys. Rev. Lett. 78, 879 (1997).

[49] N. J. Fisch and J. M. Rax, Phys. Rev. Lett. 69, 612 (1992). 
FIG. 1. Transversal dynamics of an electron in ion channel without interaction with laser pulse. Straight line 1 is the electron trajectory without angular momentum $(L=0)$. Circumference 2 is the electron trajectory with maximal or minimal angular momentum $(L=I$ or $L=-I)$. Curve 3 is the electron trajectory with $(L=I / 2)$.

FIG. 2. The dependence of $Q$ on $X$ for linear absorption regime at $\lambda=5$.

FIG. 3. A sequence of snapshots of phase space of the electron moving across the laser pulse illustrating the heating in the strongly nonlinear regime. The thin lines are surfaces of constant Hamiltonian. The particles are indicate by the heavy lines. They are first pulled through the hyperbolic fixed point from below separatrix [(a)-(c)]. Half of the particles are expelled above separatrix $[(\mathrm{d})-(\mathrm{e})]$. Parameters are $\epsilon=0.1, A_{0}=1.5, p_{0}=3.9, p_{\perp, 0}=2$, $C_{2}=0$.

FIG. 4. Schematic of the electron acceleration in the frame of the laser pulse. Electron first is pre-accelerated in the front of laser pulse but have velocity less than group velocity of laser pulse. In the frame of laser pulse the electron moves across the laser pulse. (The electron is overtaken by the laser pulse in laboratory frame).

FIG. 5. Typical electron trajectory of an electrons moving across the laser pulse in the strongly nonlinear regime without energy gain ( $a, b)$ and with energy gain $(\mathrm{c}, \mathrm{d})$. Parameters are $\epsilon=0.12, A_{0}=3, p_{0}=5.9, p_{\perp, 0}=2$, $C_{2}=0$.

FIG. 6. The dynamics of the Hamiltonian during the interaction of an electron with laser pulse with energy gain (a) and without energy (b). Parameters are the same as for Fig. 5. 


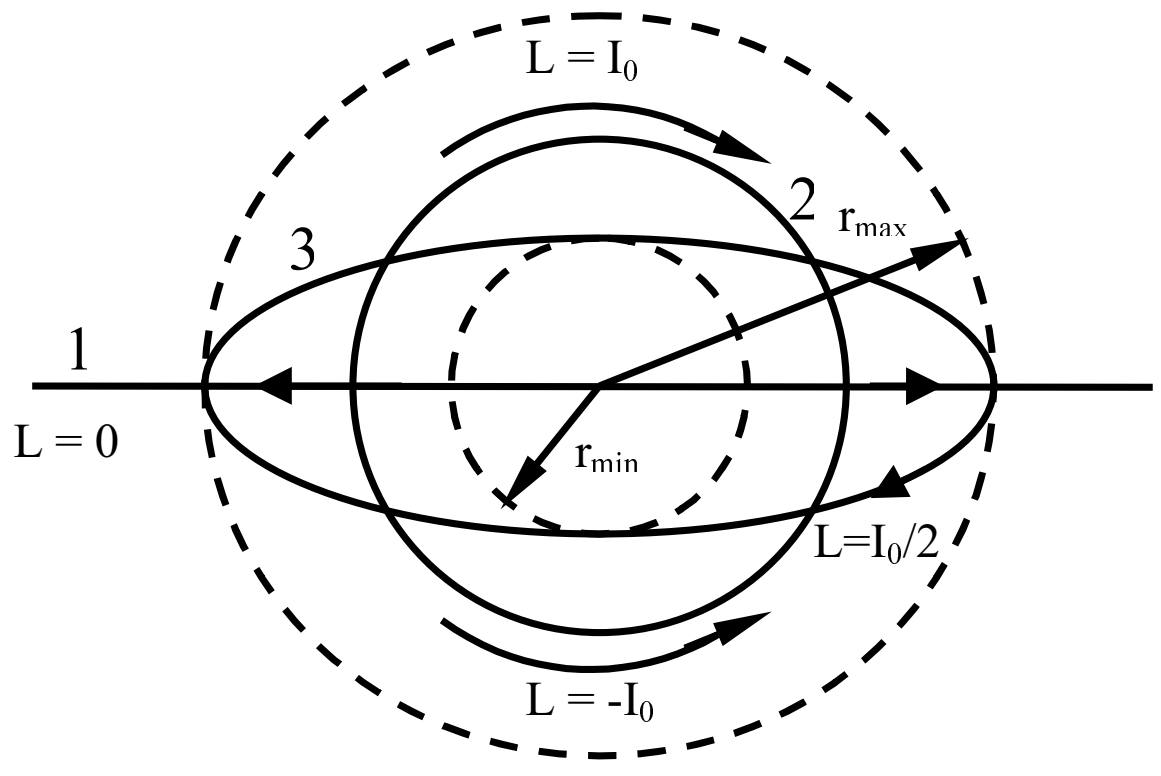




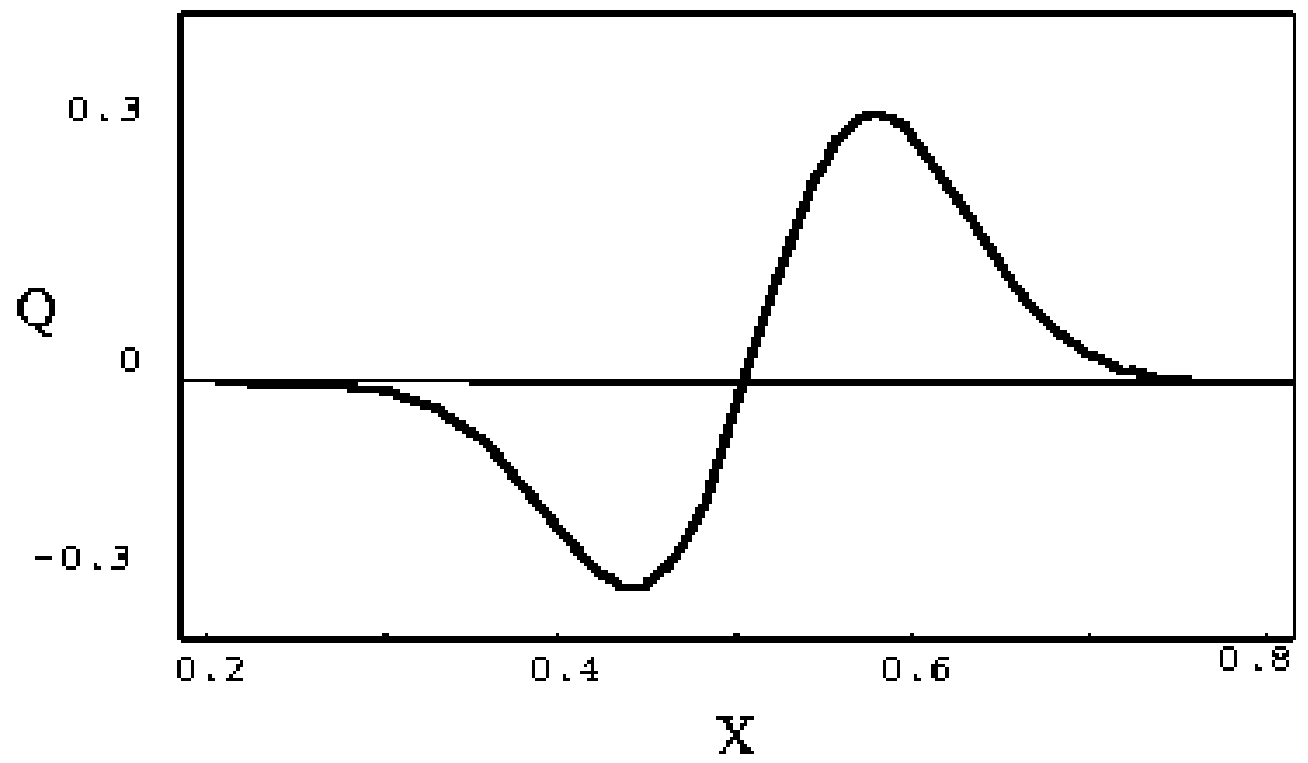




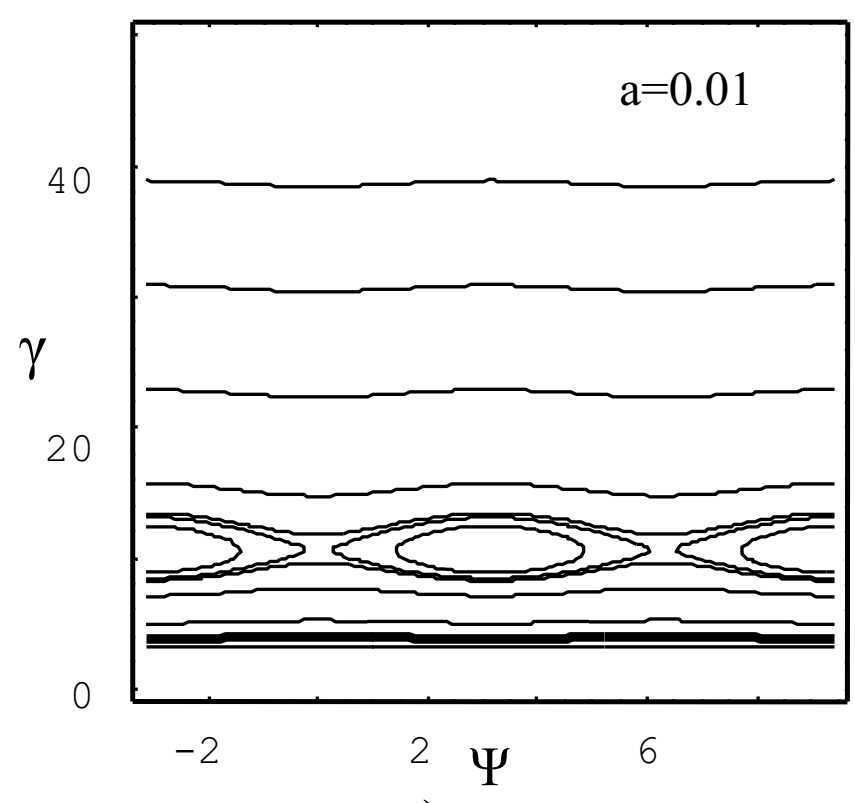

a)

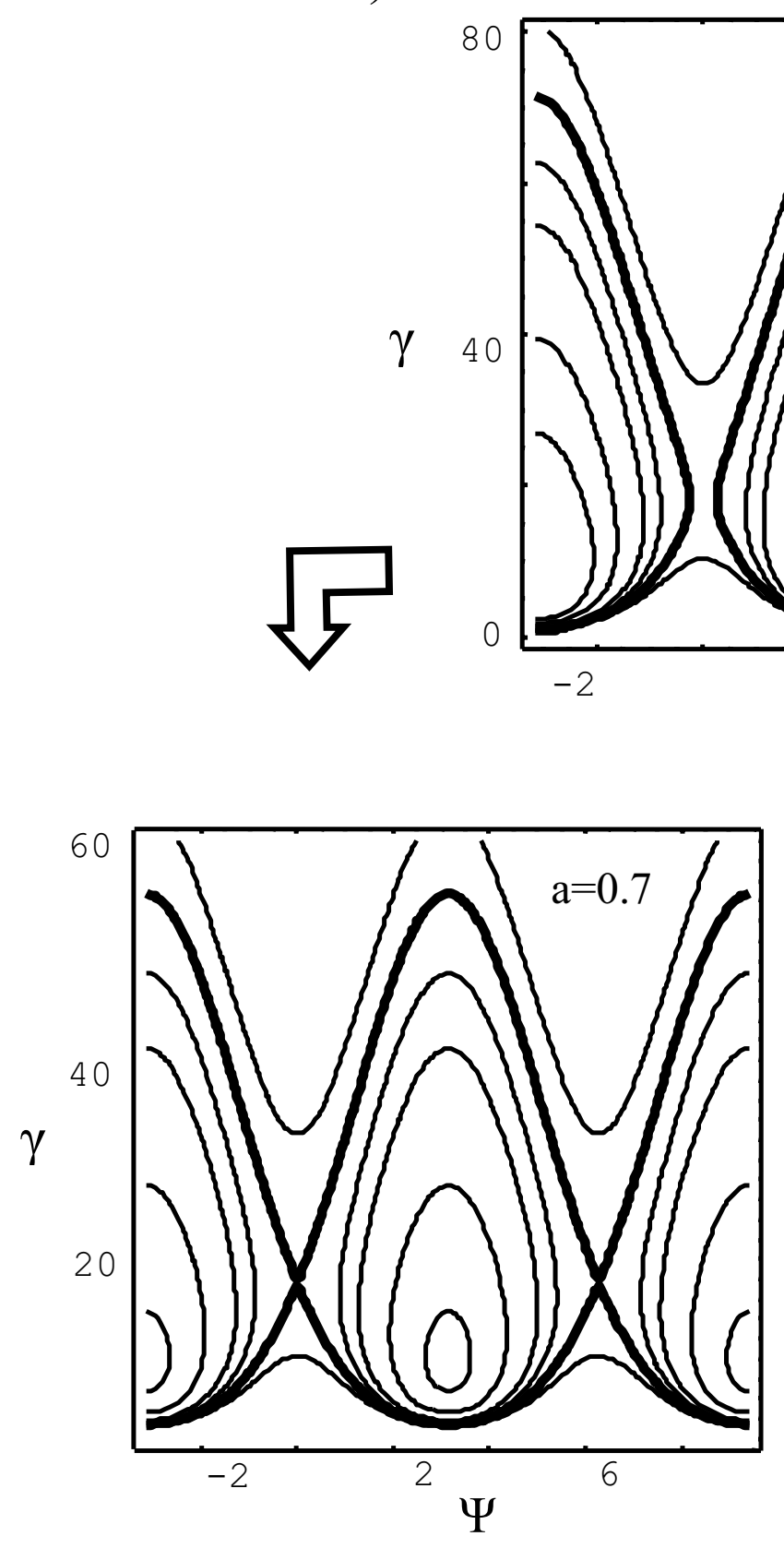

d)

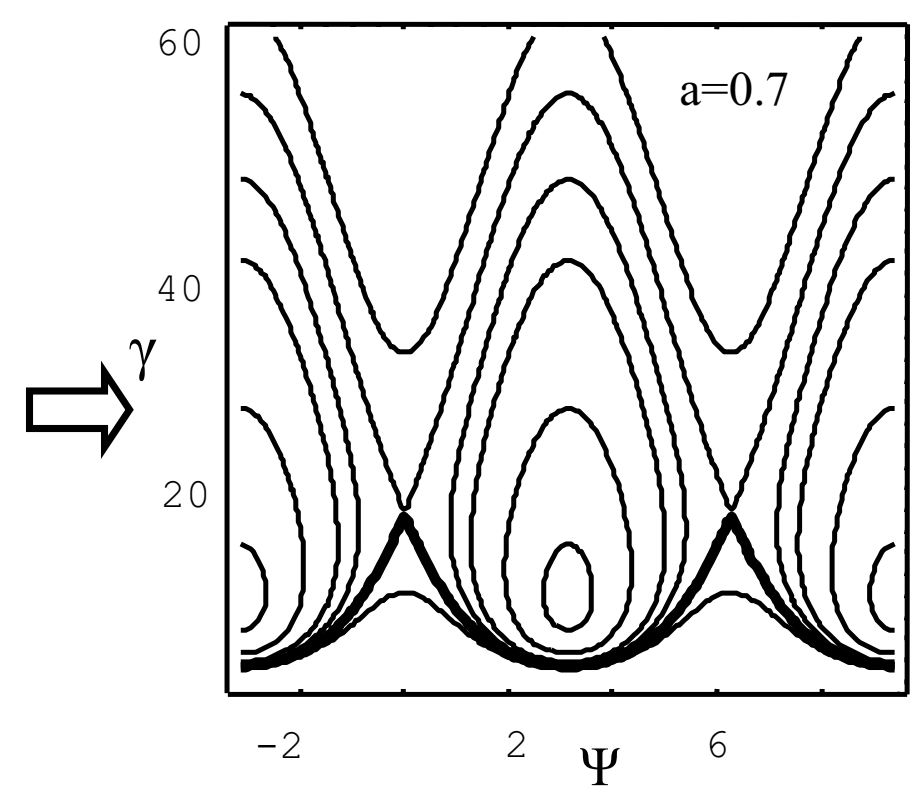

b)

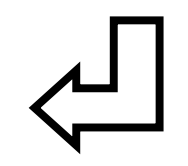

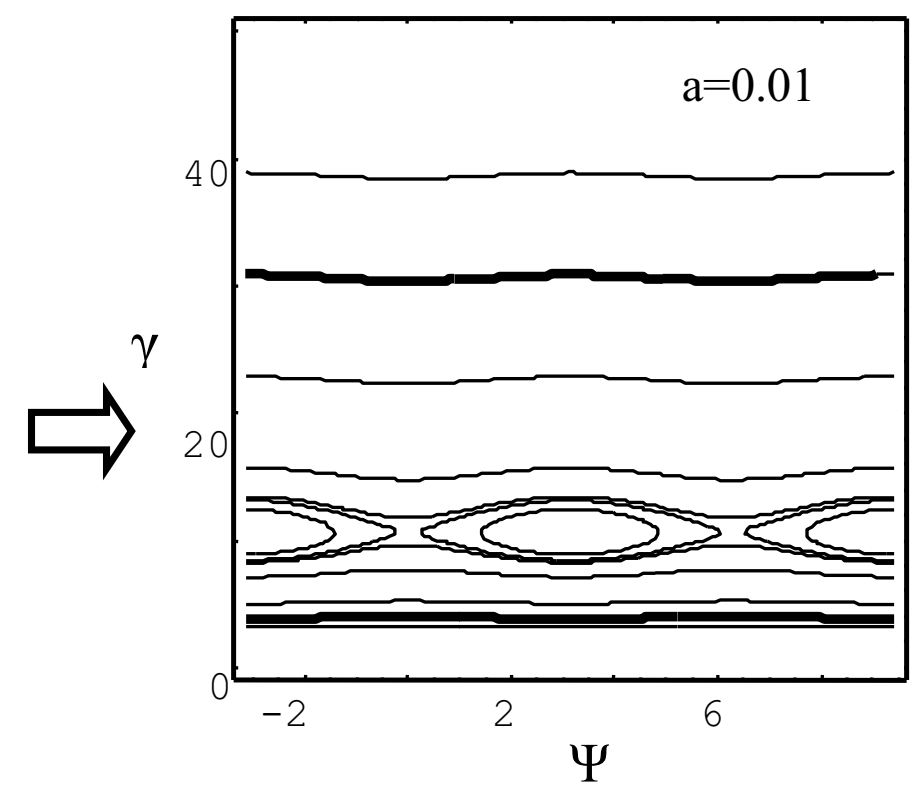

e) 

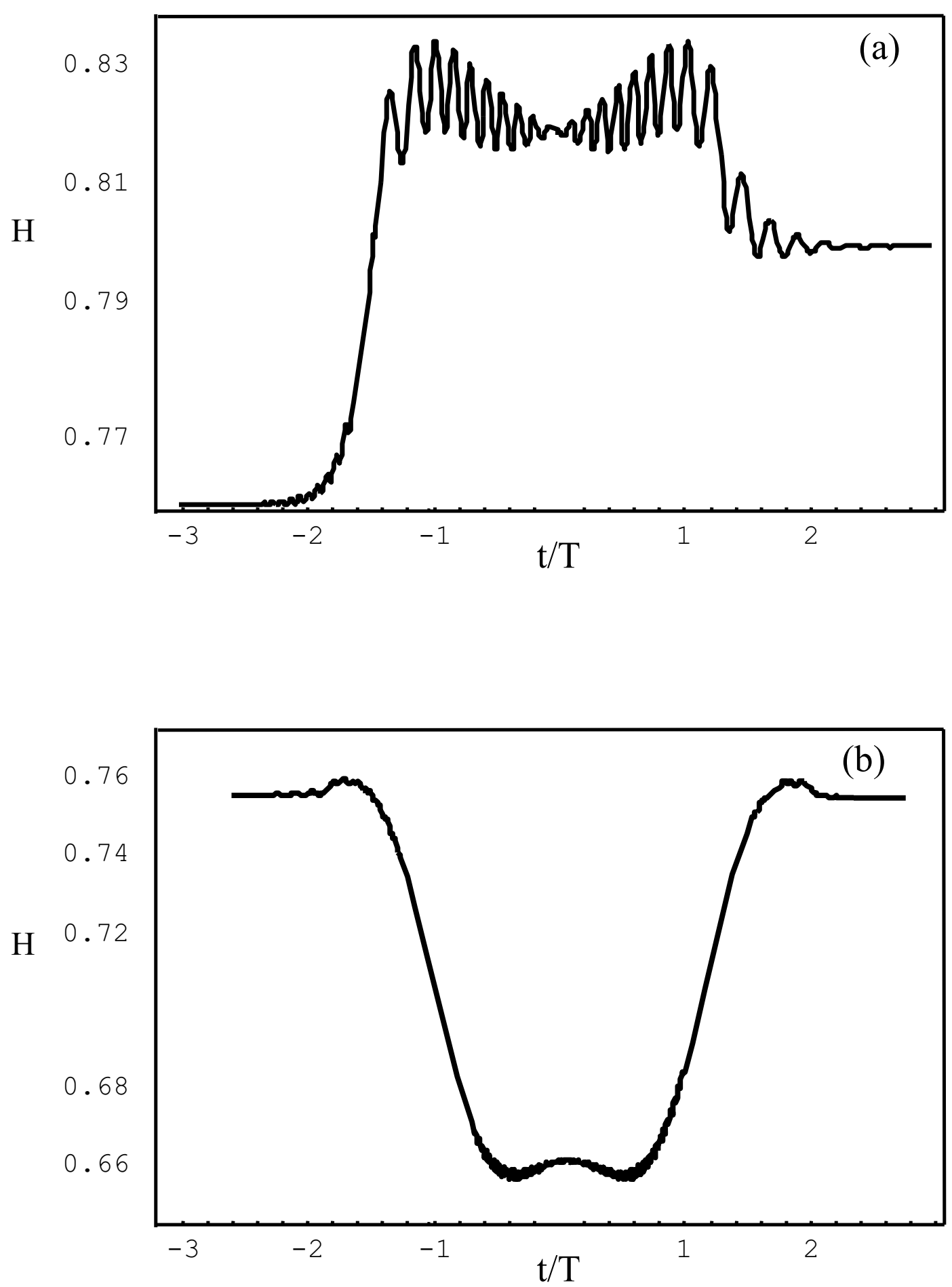

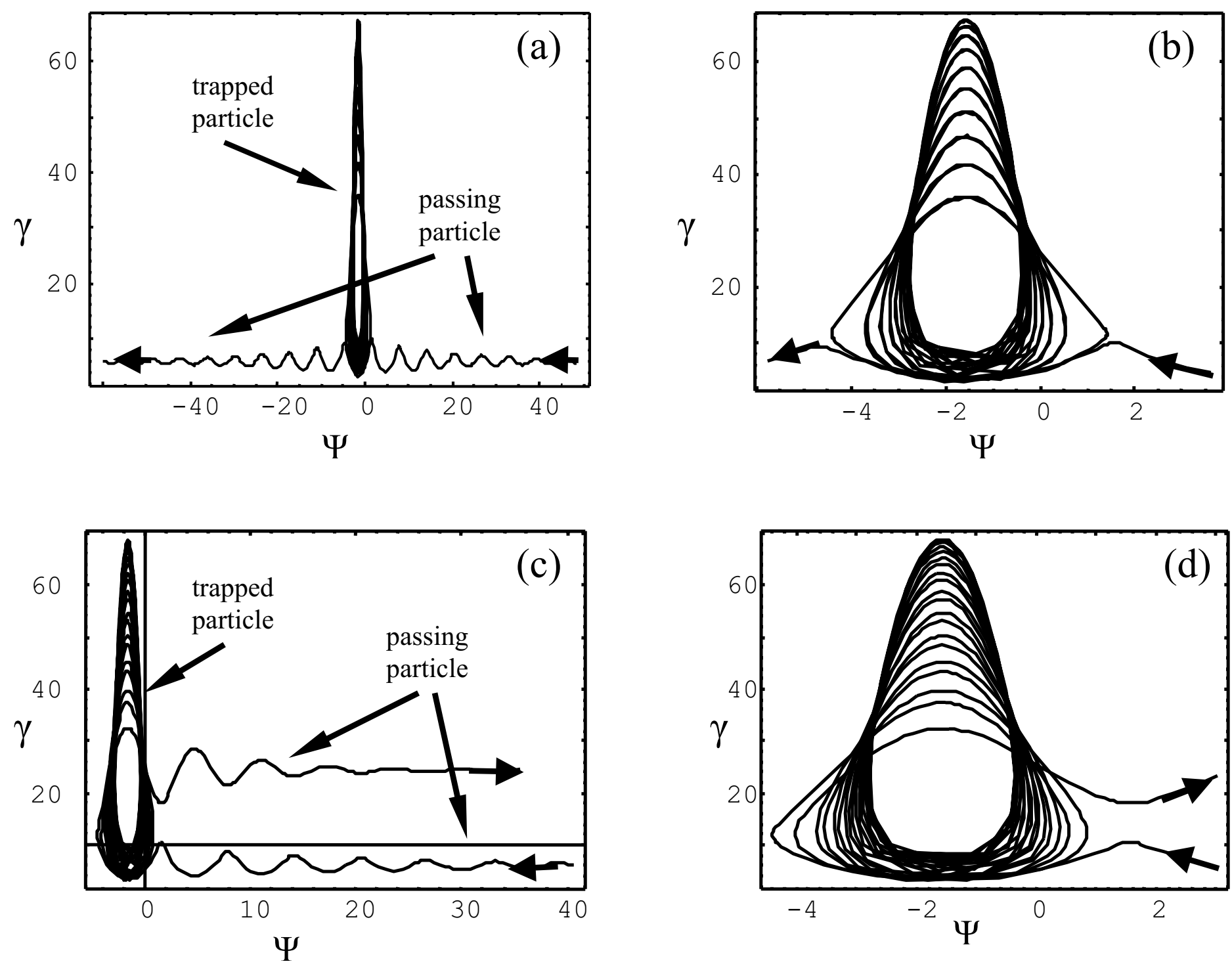


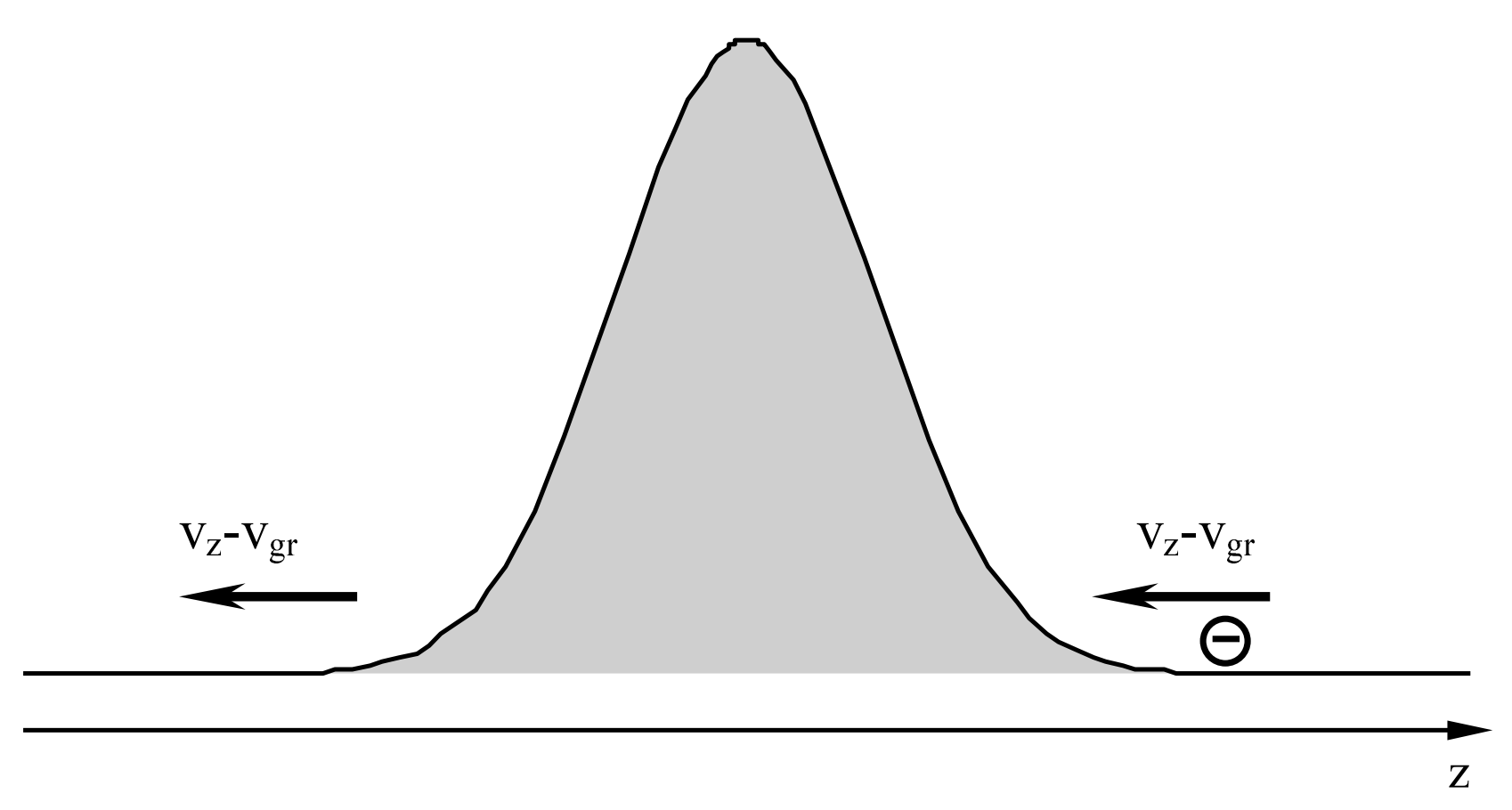




\section{External Distribution}

Plasma Research Laboratory, Australian National University, Australia

Professor I.R. J ones, Flinders University, Australia

Professor J oão Canalle, Instituto de Fisica DEQ/IF - UERJ , Brazil

Mr. Gerson O. Ludwig, Instituto Nacional de Pesquisas, Brazil

Dr. P.H. Sakanaka, Instituto Fisica, Brazil

The Librarian, Culham Laboratory, England

Library, R61, Rutherford Appleton Laboratory, England

Mrs. S.A. Hutchinson, JET Library, England

Professor M.N. Bussac, Ecole Polytechnique, France

Librarian, Max-Planck-Institut für Plasmaphysik, Germany

J olan Moldvai, Reports Library, MTA KFKI-ATKI, Hungary

Dr. P. Kaw, Institute for Plasma Research, India

Ms. P.J . Pathak, Librarian, Insitute for Plasma Research, India

Ms. Clelia De Palo, Associazione EURATOM-ENEA, I taly

Dr. G. Grosso, Instituto di Fisica del Plasma, Italy

Librarian, Naka Fusion Research Establishment, J AERI, J apan

Library, Plasma Physics Laboratory, Kyoto University, J apan

Research Information Center, National Institute for Fusion Science, J apan

Dr. O. Mitarai, Kyushu Tokai University, J apan

Library, Academia Sinica, Institute of Plasma Physics, People's Republic of China

Shih-Tung Tsai, Institute of Physics, Chinese Academy of Sciences, People's Republic of China

Dr. S. Mirnov, TRINITI, Troitsk, Russian Federation, Russia

Dr. V.S. Strelkov, Kurchatov Institute, Russian Federation, Russia

Professor Peter Lukac, Katedra Fyziky Plazmy MFF UK, Mlynska dolina F-2, Komenskeho Univerzita, SK-842 15 Bratislava, Slovakia

Dr. G.S. Lee, Korea Basic Science Institute, South Korea

Mr. Dennis Bruggink, Fusion Library, University of Wisconsin, USA

Institute for Plasma Research, University of Maryland, USA

Librarian, Fusion Energy Division, Oak Ridge National Laboratory, USA

Librarian, Institute of Fusion Studies, University of Texas, USA

Librarian, Magnetic Fusion Program, Lawrence Livermore National Laboratory, USA

Library, General Atomics, USA

Plasma Physics Group, Fusion Energy Research Program, University of California at San Diego, USA

Plasma Physics Library, Columbia University, USA

Alkesh Punjabi, Center for Fusion Research and Training, Hampton University, USA

Dr. W.M. Stacey, Fusion Research Center, Georgia Institute of Technology, USA

Dr. J ohn Willis, U.S. Department of Energy, Office of Fusion Energy Sciences, USA

Mr. Paul H. Wright, Indianapolis, Indiana, USA 
The Princeton Plasma Physics Laboratory is operated by Princeton University under contract with the U.S. Department of Energy.

\author{
Information Services \\ Princeton Plasma Physics Laboratory \\ P.O. Box 451 \\ Princeton, NJ 08543
}

Phone: 609-243-2750

Fax: 609-243-2751

e-mail: pppl_info@pppl.gov

Internet Address: http://www.pppl.gov 